\title{
SYMPLECTIC QUANDLES AND PARABOLIC REPRESENTATIONS OF 2-BRIDGE KNOTS AND LINKS
}

\author{
KYEONGHEE JO AND HYUK KIM
}

\begin{abstract}
In this paper we study the parabolic representations of 2-bridge links by finiding arc coloring vectors on the Conway diagram. The method we use is to convert the system of conjugation quandle equations to that of symplectic quandle equations. In this approach, we have an integer coefficient monic polynomial $P_{K}(u)$ for each 2-bridge link $K$, and each zero of this polynomial gives a set of arc coloring vectors on the diagram of $K$ satisfying the system of symplectic quandle equations, which gives an explicit formula for a parabolic representation of $K$. We then explain how these arc coloring vectors give us the closed form formulas of the complex volume and the cusp shape of the representation. As other applications of this method, we show some interesting arithmetic properties of the Riley polynomial and of the trace field, and also describe a necessary and sufficient condition for the existence of epimorphisms between 2-bridge link groups in terms of divisibility of the corresponding Riley polynomials.
\end{abstract}

\section{Contents}

1. Introduction 1

2. Preliminaries 4

3. Symplectic quandle structure on the set of parabolic elements in $S L(2, \mathbb{C})$

4. Parabolic representations of 2-bridge knots and links 10

5. Trace field 25

6. Complex volume and Cusp shape 27

7. Epimorphisms between knot groups 30

Appendix A. Torus knots and links 36

Appendix B. $J\left(2 n_{1}, 2 n_{2}, \cdots, 2 n_{m}\right)$-knots and links 38

References 39

\section{INTRODUCTION}

Since the volume conjecture connects the quantum invariants of a knot and the hyperbolic geometry of the knot complement, it has attracted a lot of attentions in the past two decades. (See for instance a recent book by Murakami and Yokota [27] for an introduction

2010 Mathematics Subject Classification. 57M25, 57M27.

Key words and phrases. 2-bridge links, parabolic representations, symplectic quandles.

The second author was supported by the National Research Foundation of Korea(NRF) grant funded by the Korea government(MSIT) (NRF-2018R1A2B6005691). 
to the subject.) Also it was further generalized by Gukov, using $S L(2, \mathbb{C})$ Chern-Simons theory, into the form of an asymptotic expansion of the colored Jones polynomial whose leading term is the complex volume and a subleading term is essentially the Reidemeister torsion [14]. The real part of the complex volume is the hyperbolic volume and the imaginary part is the Chern-Simons invariant, which are very important invariants of hyperbolic 3 -manifolds but in general difficult to compute. The simplicial formula of these invariants using ideal triangulations of hyperbolic 3-manifolds was set by Neumann [30], and then more efficient way in the cusped case was given by Zickert using a relative version of the earlier theory [40].

The method of Neumann and Zickert can be applied to the link complement, and a diagrammatic method using quandle homology was studied by Inoue and Kabaya [18]. And then Cho and Murakami introduced a combinatorial way of computing the complex volume motivated from the volume conjecture [6, 7] following Yokota's work [39]. In fact they explicitly expressed the complex volume formula in the form of a state sum for a given link diagram, signifying its origin in quantum invariants, using region variables (or called $w$-variables), which can be obtained from quandle coloring vectors. This formula is by far the simplest way of describing the complex volume from a diagram of a link in a closed form formula in terms of region variables. The notion of complex volume was defined for hyperbolic manifolds, but can be generalized to any $S L(2, \mathbb{C})$ representation and we are using this generalized version in this paper. (See [40] for the complex volume of a representation and [13] for the $S L(n, \mathbb{C})$ case.) For actual computations of the volume, one has to solve a system of algebraic equations, which essentially corresponds to the hyperbolicity equations, to get the region variables [22]. But this system of equations is not easy to solve in general, and instead, one solves for the quandle equations to get the coloring vectors in $\mathbb{C}^{2}$, which then gives the region variables immediately by taking the determinant of the coloring vectors with a generic fixed vector [6, 7]. Therefore having an explicit volume formula is essentially the same as an explicit formula for the arc coloring vectors by Cho-Murakami's result, and we describe how to get all these for 2-bridge links in this paper.

Also this coloring vector is defined on each arc of the link diagram and is nothing but a short hand notation for a parabolic element in $S L(2, \mathbb{C})$ (see Section 3), giving an explicit description of a parabolic representation of Wirtinger generators, and the volume above is the volume of this representation. Once we have a $\operatorname{PSL}(2, \mathbb{C})$ representation, we have a pseudo-hyperbolic structure [22, 35] and can talk about hyperbolic invariants such as complex volume, and another such invariant is a cusp shape. This of course can be obtained by calculating the longitude algebraically, but can also be obtained using $w$-variables in the form of an explicit state sum formula just like the complex volume. And hence the cusp shape of a parabolic representation also can be obtained once we have a set of arc coloring vectors. (See Section 6.)

Therefore the problem of computing all these quantities boils down to computing arc coloring vectors, which essentially is to find an efficient way of solving the system of conjugation equations determined by Wirtinger relators. In general the computation for solving conjugation quandle is very complicated; even the cases for 8-crossing knots do not seem to 
be completely settled. And one of the main purpose of this paper is to find an algorithm by "linearizing" the system of conjugation equations. If we inspect carefully, we can see that solving this conjugation equation reduces to solving a symplectic quandle equation, which is semi-linear in the sense that linear in one variable and quadratic in the other variable at each crossing. The notion of the symplectic quandle was introduced in [29] and [38], but the relation between conjugation and symplectic quandle doesn't seem to be considered before. This change gives us much advantage in carrying out the actual computations as well as conceptual approaches. In this quandle formulation, the determinant of two coloring vectors, called a symplectic form, appears naturally, and this plays an important role in this paper (and this also appears in other papers in different contexts) and will be called a $u$-variable in contrast to a $w$-variable.

When we apply the above argument to 2-bridge links, especially in the Conway form, we can solve the symplectic quandle equation and obtain an explicit formula for the arc coloring vectors in terms of $u:=\langle a, b\rangle:=\operatorname{det}(a, b)$, where $a$ and $b$ are two initial coloring vectors at the two bridges. It turns out that the solution $u$ is obtained as zeros of one single integer coefficient monic polynomial $P(u)$, called the "rep-polynomial" in this paper following Riley [32]. Then the solution gives the arc coloring vectors and hence the region variables immediately, and then the complex volume from the formula of Cho-Murakami and the cusp shape from Kim-Kim-Yoon [23]. Here everything is concrete and explicit and can be given in an exact form.

Needless to say, this polynomial $P(u)$ should be related to the well known Riley polynomial $R(y)$, and indeed it turns out that $\frac{1}{u} P(u)= \pm R\left(u^{2}\right)$. By deriving $P(u)$ from the symplectic quandle, we do not just recover the famous old result back and the coloring vectors, but also we found some interesting unknown arithmetic properties of the Riley polynomial and of the trace field: Namely splitting of the Riley polynomial for the knot case, $R\left(u^{2}\right)= \pm g(u) g(-u)$, for some integral coefficient polynomial $g(u)$ (this remarkable property reminds us Hirasawa-Murasugi conjecture [15]) as well as the fact that the trace field is generated by $u$ instead of $y$. Note that $u$ is a square root of $y$.

One good point of using Conway form instead of Schubert form is to turn the diagram upside down to see another two generators of the knot group $G(K)$. We can get the corresponding rep-polynomial $P^{\prime}(u)$ which has an equal right as $P(u)$, and this observation gives us an interesting application in the epimorphism problem. In the knot theory, the epimorphism problem has been studied quite extensively and for the 2-bridge knot case, the problem of characterizing the epimorphism pair, $G(K) \rightarrow G\left(K^{\prime}\right)$ in terms of Conway form is essentially settled down by Ohtsuki-Riley-Sakuma [31] and Aimi-Lee-Sakai-Sakuma [2]. (See also [36].) Recently Kitano and Morifuji proved the existence of an epimorphism when the Riley polynomial, (and hence the rep-ploynomial) of $K^{\prime}$ divides that of $K$ [24]. And we show that the converse also holds if we allow both rep-polynomials $P(u)$ and $P^{\prime}(u)$ in the divisibility, and show that we can generalize to a similar statement for the link case also. (See Section 7.)

The paper is organized as follows. We first setup the symplectic quandle equations and describe the arc coloring vectors as solutions of the equations in Sections 2 and 3, and then discuss $u$-variables and rep-polynomials in Section 4. Then as applications of this 
approach, we discuss the arithmetic properties mentioned above in Section 5, the complex volume and cusp shape in Section 6, and then the epimorphism problem in Section 7. In appendix we present some explicit formulas of the arc coloring vectors using Chebyshev polynomials.

\section{Preliminaries}

2.1. 2-bridge Knots and Links. There are two famous descriptions for 2-bridge knots or links, Scubert's normal form and Conway's normal form. Together, a knot and link are called a link in this paper, unless we need to specify it.

Scubert's normal form Each 2-bridge link has an associated pair of coprime integers $(\alpha, \beta)$ where $\alpha$ is positive and $\beta$ is an odd integer such that $-\alpha<\beta<\alpha$. We denote the knot or link by $S(\alpha, \beta)$ and call it Schubert's normal form. The followings are well-known facts (see [3] or [21] for more details).

- $S(\alpha, \beta)$ is a knot if $\alpha$ is odd and a 2-component link if $\alpha$ is even.

- The mirror of $S(\alpha, \beta)$ is equivalent to $S(\alpha,-\beta)$.

- $S(\alpha, \beta)$ and $S\left(\alpha^{\prime}, \beta^{\prime}\right)$ are equivalent as unoriented knots or links if and only if

$$
\alpha=\alpha^{\prime}, \beta^{\prime} \equiv \beta^{ \pm 1} \quad(\bmod \alpha) .
$$

Note that if we consider a knot (or a link) and its mirror as equivalent, $S(\alpha, \beta)$ and $S\left(\alpha^{\prime}, \beta^{\prime}\right)$ are equivalent knots or links if and only if

$$
\alpha=\alpha^{\prime}, \beta^{\prime} \equiv \pm \beta^{ \pm 1} \quad(\bmod \alpha) \text {. }
$$

Conway's normal form Each 2-bridge link has an associated sequence of non-zero integers $n_{1}, n_{2}, \cdots, n_{k}$, as indicated in Figure 8, where $\left|n_{j}\right|$ is the number of crossing contained in the $j$ th block and its sign $\epsilon_{j}=\frac{n_{j}}{\left|n_{j}\right|}$ is defined as follows : $\epsilon_{2 i+1}=1$ or $\epsilon_{2 i}=-1$ if the twists of the block are right-handed and $\epsilon_{2 i+1}=-1$ or $\epsilon_{2 i}=1$ if they are left-handed. We denote the unoriented 2-bridge link with this regular projection by $C\left[n_{1}, n_{2}, \cdots, n_{k}\right]$, which is called Conway's normal form. For example, $C[3,2,3]$ is shown in Figure 17. (See [10] or [20].)

Note that such a diagram of a 2-bridge link $C\left[n_{1}, n_{2}, \cdots, n_{k}\right]$ corresponds to a rational tangle with slope

$$
\left[n_{1}, n_{2}, \cdots, n_{k}\right]:=\frac{1}{n_{1}+\frac{1}{n_{2}+\frac{1}{\cdot \frac{1}{n_{k-1}+\frac{1}{n_{k}}}}}} .
$$

Let $\alpha(>0)$ and $\beta$ be coprime integers obtained by the slope of $C\left[n_{1}, n_{2}, \cdots, n_{k}\right]$, that is,

$$
\frac{\beta}{\alpha}=\left[n_{1}, n_{2}, \cdots, n_{k}\right]
$$

Then it is well-known that $C\left[n_{1}, n_{2}, \cdots, n_{k}\right]$ is equivalent to $S(\alpha, \beta)$. Furthermore each 2-bridge link $S(\alpha, \beta)$ in Schubert's normal form can be deformed into Conway's normal form $C\left[n_{1}, n_{2}, \cdots, n_{k}\right]$ uniquely, if we require that all the $n_{i}^{\prime} s$ are either positive or negative 
and $\left|n_{k}\right| \neq 1$. We will call the unique Conway's normal form the cannonical Conway's normal form of $S(\alpha, \beta)$.

The following notation will also be used in this paper :

$$
J\left(n_{1}, n_{2}, n_{3}, \cdots, n_{k}\right)=: C\left[n_{1},-n_{2}, n_{3}, \cdots,(-1)^{k+1} n_{k}\right] .
$$

That is, $n_{i}$ is always positive whether $i$ is odd or not if the twists of the $i$-th block are right-handed, and negative if the twists are left-handed in $J\left(n_{1}, n_{2}, n_{3}, \cdots, n_{k}\right)$-notation.

Notice that $C\left[-n_{1},-n_{2}, \cdots,-n_{k}\right]$ is the mirror of $C\left[n_{1}, n_{2}, \cdots, n_{k}\right]$ and

$$
C\left[(-1)^{k+1} n_{k},(-1)^{k+1} n_{k-1}, \cdots,(-1)^{k+1} n_{1}\right]
$$

is the upside-down of $C\left[n_{1}, n_{2}, \cdots, n_{k}\right]$, i.e., the diagram obtained by a half rotation with respect to the horizontal axis. It is well-known that if $\frac{\beta}{\alpha}=\left[n_{1}, n_{2}, \cdots, n_{k}\right]$, then

$$
\left[(-1)^{k+1} n_{k},(-1)^{k+1} n_{k-1}, \cdots,(-1)^{k+1} n_{1}\right]=\frac{\beta^{\prime}}{\alpha}, \beta \beta^{\prime} \equiv 1(\bmod \alpha) .
$$

2.2. Riley polynomials of 2-bridge links. For a knot or link $K$, the fundamental group of the complement is called the knot group or the link group and is denoted by $G(K)$.

The knot group of a 2-bridge knot $K=S(\alpha, \beta)$ always has a presentation of the form

$$
G(K)=\pi_{1}\left(\mathrm{~S}^{3} \backslash K\right)=\langle a, b \mid w a=b w\rangle,
$$

where $w$ is of the form

$$
w=a^{\epsilon_{1}} b^{\epsilon_{2}} a^{\epsilon_{3}} b^{\epsilon_{4}} \cdots a^{\epsilon_{\alpha-2}} b^{\epsilon_{\alpha-1}}
$$

with each $\epsilon_{i}=-(-1)^{\left[i \frac{\beta}{\alpha}\right]}$ a Note that $\epsilon_{i}=\epsilon_{\alpha-i}$ and the generators $a$ and $b$ come from the two bridges and represent the meridians.

Suppose that $\rho: G(K) \rightarrow S L(2, \mathbb{C})$ is a non-abelian parabolic representation, i.e., the trace of $\rho$-image of any meridian is 2 . Then after conjugating if necessary, we may assume

$$
\rho(a)=\left(\begin{array}{ll}
1 & 1 \\
0 & 1
\end{array}\right) \quad \text { and } \quad \rho(b)=\left(\begin{array}{cc}
1 & 0 \\
-y & 1
\end{array}\right) .
$$

Riley had shown in 32 that $y$ determines a non-abelian parabolic representation if and only if $W_{11}=0$. Here $W_{i j}$ is the $(i, j)$-element of

$$
\begin{aligned}
W & =\rho(w)=\rho(a)^{\epsilon_{1}} \rho(b)^{\epsilon_{2}} \rho(a)^{\epsilon_{3}} \rho(b)^{\epsilon_{4}} \cdots \rho(a)^{\epsilon_{\alpha-2}} \rho\left((b)^{\epsilon_{\alpha-1}}\right. \\
& =\left(\begin{array}{ll}
1 & 1 \\
0 & 1
\end{array}\right)^{\epsilon_{1}}\left(\begin{array}{cc}
1 & 0 \\
-y & 1
\end{array}\right)^{\epsilon_{2}}\left(\begin{array}{ll}
1 & 1 \\
0 & 1
\end{array}\right)^{\epsilon_{3}} \cdots\left(\begin{array}{ll}
1 & 1 \\
0 & 1
\end{array}\right)^{\epsilon_{\alpha-2}}\left(\begin{array}{cc}
1 & 0 \\
-y & 1
\end{array}\right)^{\epsilon_{\alpha-1}},
\end{aligned}
$$

for $i, j=1,2$. Furthermore $W_{11}$ has no repeated roots and the non-abelian parabolic representations bijectively correspond to the roots of the polynomial $W_{11} \in \mathbb{Z}[y]$, the Riley polynomial. Note that the degree of $W_{11}$ is $\frac{1}{2}(\alpha-1)$.

For the case of 2-bridge link $K=S(\alpha, \beta)$,

$$
G(K)=\left\langle a, b \mid w b=b w, w^{*} a=a w^{*}\right\rangle,
$$

\footnotetext{
aThis definition coincides with Riley's definition in 32 .
} 
where $w$ is given as

$$
w=a^{\epsilon_{1}} b^{\epsilon_{2}} a^{\epsilon_{3}} b^{\epsilon_{4}} \cdots b^{\epsilon_{\alpha-2}} a^{\epsilon_{\alpha-1}}
$$

and $w^{*}$ as

$$
w^{*}=b^{\epsilon_{1}} a^{\epsilon_{2}} b^{\epsilon_{3}} a^{\epsilon_{4}} \cdots a^{\epsilon_{\alpha-2}} b^{\epsilon_{\alpha-1}} .
$$

Then by following Riley's argument about the knot cases, one can prove that $W_{12}=-\frac{1}{y} W_{21}^{*}$, and the non-zero roots of $W_{12}$ correspond to non-abelian parabolic representations of $K$ from $\rho(w) \rho(b)=\rho(b) \rho(w)$ and $\rho\left(w^{*}\right) \rho(a)=\rho(a) \rho\left(w^{*}\right)$. For this reason, we will call the polynomial $W_{12} \in \mathbb{Z}[y]$ the Riley polynomial of a link $K$ in this paper. Note that the degree of $W_{12} \in \mathbb{Z}[y]$ is $\frac{1}{2}(\alpha-2)$.

We use the notation $\mathcal{R}(y)$ to denote the Riley polynomial so that $\mathcal{R}(y)=W_{11}(y)$ if $K$ is a knot and $\mathcal{R}(y)=W_{12}(y)$ if $K$ is a link. See also section 4.4 .

2.3. Chebyshev polynomials. Chebyshev polynomials, which are defined by a threeterm recursion

$$
g_{n+1}(t)=t g_{n}(t)-g_{n-1}(t)
$$

can be used to describe some properties and characteristics of knots and links. We denote the Chebyshev polynomials $g_{n}(t)$ with the intial condition $g_{0}(t)=a, g_{1}(t)=b$ by $C h_{n}^{t}(a, b)$, which clearly depends on the initial condition linearly. And the following properties are also obvious:

$$
\begin{aligned}
& C h_{n}^{t}(a, b)=C h_{n-k}^{t}\left(C h_{k}^{t}(a, b), C h_{k+1}^{t}(a, b)\right) \\
& C h_{n}^{t}(a, b)=a C h_{n}^{t}(1,0)+b C h_{n}^{t}(0,1)
\end{aligned}
$$

If we denote a particular Chebyshev polynomials $C h_{n}^{t}(0,1)$ by $p_{n}(t)$, then

$$
C h_{n}^{t}(1,0)=C h_{n-1}^{t}(0,-1)=-C h_{n-1}^{t}(0,1)=-p_{n-1}(t)
$$

and arbitrary Chebyshev polynomials are expressed as linear combinations of $p_{n-1}(t)$ and $p_{n}(t)$ as follows :

$$
C h_{n}^{t}(a, b)=-a p_{n-1}(t)+b p_{n}(t)
$$

Sometimes we will call such Chebyshev polynomials $t$-Chebyshev polynomials when it is needed to specify the variable $t$. The following Chebyshev polynomials are frequently used:

$$
\begin{aligned}
& f_{n}(t):=p_{n+1}(t)-p_{n}(t)=C h_{n+1}^{t}(1,1)=C h_{n}^{t}(1, t-1) \\
& v_{n}(t):=p_{n+1}(t)-p_{n-1}(t)=C h_{n}^{t}(2, t)
\end{aligned}
$$

The following properties for Chebyshev polynomials are well-known or easily proved. See [16, 34, 37] for references.

Lemma 2.1. Let $p_{n}, f_{n}, v_{n}$ be as above. Then

(i) $p_{-n}(t)=-p_{n}(t), \quad p_{n}(-t)=(-1)^{n+1} p_{n}(t)$

(ii) $p_{n}(2)=n, \quad p_{2 n}(0)=0, \quad p_{2 n+1}(0)=(-1)^{n}$

(iii) $p_{2 n+1}(t)=p_{n}\left(v_{2}(t)\right)+p_{n+1}\left(v_{2}(t)\right)=p_{n+1}(t)^{2}-p_{n}(t)^{2}$

(iv) $p_{n}(t)^{2}-p_{n-1}(t) p_{n+1}(t)=1$ 
(v) $p_{n+1}(t)^{2}-t p_{n}(t) p_{n+1}(t)+p_{n}(t)^{2}=1$

(vi) $p_{n m}(t)=p_{n}\left(v_{m}(t)\right) p_{m}(t)$

(vii) $(-1)^{n} f_{n}(-t)=p_{n}(t)+p_{n+1}(t)$

(viii) $f_{n}\left(-v_{2 k}(t)\right)=f_{n}\left(v_{k}(t)\right) f_{n}\left(-v_{k}(t)\right)$

Lemma 2.2. Let $M \in S L(2, \mathbb{C})$ and $t=\operatorname{tr} M$. Then

(i) $M^{n}=C h_{n}^{t}(I, M)=p_{n}(t) M-p_{n-1}(t) I$

(ii) $\operatorname{tr}\left(M^{n}\right)=v_{n}(t)$

\subsection{Quandle.}

Definition 2.3. A set $X$ that has a binary operation $\triangleright: X \times X \rightarrow X$ is called a quandle if the following three axioms hold:

(i) for any $a \in X, a \triangleright a=a$;

(ii) for each $a, b \in X$, there is a unique $c \in X$ such that $c \triangleright b=a$;

(iii) for each $a, b, c \in X,(a \triangleright b) \triangleright c=(a \triangleright c) \triangleright(b \triangleright c)$.

Axiom (ii) implies that quandle operation $\triangleright$ has a dual quandle operation $\triangleright^{-1}$ such that $c=a \triangleright^{-1} b$. Note that

$$
\left(a \triangleright^{-1} b\right) \triangleright b=a \quad \text { and } \quad(a \triangleright b) \triangleright^{-1} b=a,
$$

and these two operations distribute over each other. See [4, 19, 38].

Any group $G$ is a quandle with respect to the operation $a \triangleright b=b^{n} a b^{-n}, a, b \in G$ for any integer $n$. For any oriented link diagram $K$, there is a quandle $Q(K)$ defined by a Wirtinger-style presentation with one generator for each arc and a relation at each crossing: Let $\mathcal{R}(K)$ be the set of over-arcs of $K$ with an orientation. Then for each crossing, we have three elements $\alpha, \beta$ and $\gamma$ of $\mathcal{R}(K)$ and the knot quandle relation between them, $\gamma=\alpha \triangleright \beta$, is defined as in Figure 1. This quandle is called a knot quandle and it is known that this quandle is a classifying invariant of knots and unsplit links in $S^{3}$ [19, 29]. Note that quandle operation is invariant under the Reidemeister moves by the quandle axioms.

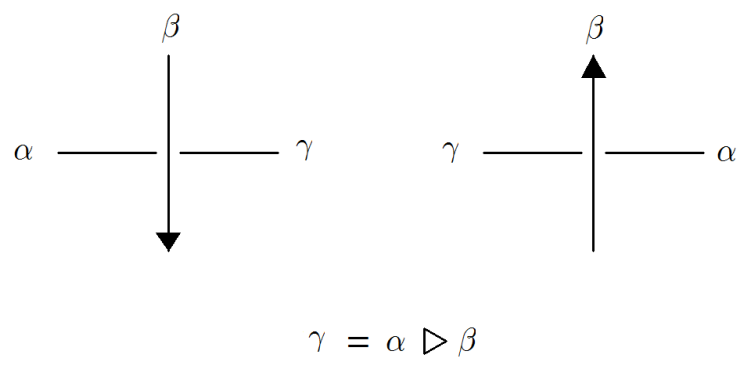

FiguRE 1. knot quandle relation

Definition 2.4. Let $X$ be a quandle, $K$ an oriented knot or link diagram. A quandle coloring $\mathcal{C}$ on $K$ is a map $\mathcal{C}: \mathcal{R}(K) \longrightarrow X$ such that

$$
\mathcal{C}(\gamma)=\mathcal{C}(\alpha) \triangleright \mathcal{C}(\beta)
$$


holds at each crossing with $\operatorname{arcs} \alpha, \beta$ and $\gamma=\alpha \triangleright \beta$. We will say that $K$ is colored by $X$ or $K$ has a $X$-coloring when there is such a quandle coloring.

\subsection{Symplectic quandle.}

Definition 2.5. Let $X$ be a finite dimensional free module over a commutative ring with identity and a non-degenerate anti-symmetric bilinear form $\langle$,$\rangle . Then (X,\langle\rangle$,$) is a quandle$ with the quandle operation

$$
x \triangleright y=x+\langle x, y\rangle y \quad \text { and } \quad x \triangleright^{-1} y=x-\langle x, y\rangle y .
$$

This type of quandle is called a symplectic quandle. See [29] for more details.

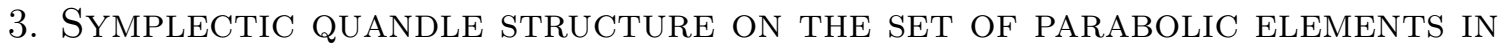

$$
S L(2, \mathbb{C})
$$

3.1. Symplectic quandle structure on $\mathbb{C}^{2}$. Let $\langle$,$\rangle be a symplectic form on \mathbb{C}^{2}$ defined by

$$
\langle x, y\rangle=\left|\begin{array}{ll}
x_{1} & y_{1} \\
x_{2} & y_{2}
\end{array}\right|=x_{1} y_{2}-x_{2} y_{1}
$$

for $x=\left(\begin{array}{l}x_{1} \\ x_{2}\end{array}\right), y=\left(\begin{array}{l}y_{1} \\ y_{2}\end{array}\right)$. Then $\left(\mathbb{C}^{2},\langle\rangle,\right)$ is a symplectic quandle with the quandle operation

$$
x \triangleright y=x+\langle x, y\rangle y .
$$

The symplectic quandle structure on $\mathbb{C}^{2}$ induces a quandle structure on the space of orbits of the action of multiplicative group $\{1,-1\}$ on $\mathbb{C}^{2}$ by scalar multilication, because negating $x$ negates $x \triangleright y$ and $x \triangleright^{-1} y$, while negating $y$ leaves them unchanged. We will denote the orbit space by $\mathfrak{C}=\mathbb{C}^{2} / \pm 1$ and call this quandle $(\mathfrak{C},\langle\rangle$,$) a (reduced) symplectic$ quandle.

3.2. Set of parabolic elements in $S L(2, \mathbb{C})$. We denote the set of parabolic elements of $S L(2, \mathbb{C})$ by $\mathcal{P}$, that is, $\mathcal{P}=\{A \in S L(2, \mathbb{C}) \mid \operatorname{tr}(A)=2\}$.

From the fact that every parabolic element is conjugate to the particular element $\left(\begin{array}{ll}1 & 1 \\ 0 & 1\end{array}\right)$, we get the following identities

$$
\begin{aligned}
A=\left(\begin{array}{ll}
a_{11} & a_{12} \\
a_{21} & a_{22}
\end{array}\right)\left(\begin{array}{ll}
1 & 1 \\
0 & 1
\end{array}\right)\left(\begin{array}{ll}
a_{11} & a_{12} \\
a_{21} & a_{22}
\end{array}\right)^{-1} & =\left(\begin{array}{cc}
1-a_{11} a_{21} & a_{11}^{2} \\
-a_{21}^{2} & 1+a_{11} a_{21}
\end{array}\right) \\
& =I+\left(\begin{array}{l}
a_{11} \\
a_{21}
\end{array}\right)\left(-a_{21}, a_{11}\right)=I+\left(\begin{array}{l}
-a_{11} \\
-a_{21}
\end{array}\right)\left(a_{21},-a_{11}\right)
\end{aligned}
$$

and

$$
A^{-1}=I-\left(\begin{array}{l}
a_{11} \\
a_{21}
\end{array}\right)\left(-a_{21}, a_{11}\right)=I-\left(\begin{array}{l}
-a_{11} \\
-a_{21}
\end{array}\right)\left(a_{21},-a_{11}\right)
$$


This gives a bijection $T$ from $\mathcal{P}$ to $\mathfrak{C}$ such that

$$
T(A)=\left[\begin{array}{l}
a_{11} \\
a_{21}
\end{array}\right]:=[a],
$$

and this map sends $A^{-1} \in \mathcal{P}$ to $[i a] \in \mathfrak{C}$, that is, $T\left(A^{-1}\right)=[i a]$. If we denote $\left(-a_{21}, a_{11}\right)$ by $\hat{a}$, then $\hat{a} b=\langle a, b\rangle$ and $A$ and $A^{-1}$ can be expressed as

$$
A=I+a \hat{a}, \quad A^{-1}=I-a \hat{a}=I+(i a)(\hat{i a}) .
$$

The following proposition shows that $T$ defines an isomorphism between the quandle $(\mathcal{P}$, conjugation) and the symplectic quandle $(\mathfrak{C},\langle\rangle$,$) .$

Proposition 3.1. If $T(A)=[a]$ and $T(B)=[b]$ then

$$
T\left(B^{-1} A B\right)=[a+\langle a, b\rangle b] \in \mathfrak{C}
$$

and

$$
T\left(B A B^{-1}\right)=[a-\langle a, b\rangle b] \in \mathfrak{C} .
$$

Proof. The first identity follows from

$$
\begin{aligned}
B^{-1} A B & =(I-b \hat{b})(I+a \hat{a})(I+b \hat{b}) \\
& =(I-b \hat{b}+a \hat{a}-b \hat{b} a \hat{a})(I+b \hat{b}) \\
& =(I-b \hat{b}+a \hat{a}-\langle b, a\rangle b \hat{a})(I+b \hat{b}) \\
& =I+a \hat{a}-\langle b, a\rangle b \hat{a}+\langle a, b\rangle a \hat{b}+\langle a, b\rangle^{2} b \hat{b} \\
& =I+a \hat{a}+\langle a, b\rangle(a \hat{b}+b \hat{a})+\langle a, b\rangle^{2} b \hat{b} \\
& =I+(a+\langle a, b\rangle b)(\hat{a}+\langle a, b\rangle \hat{b}) \\
& =I+c \hat{c}
\end{aligned}
$$

where $c=a+\langle a, b\rangle b$ by obvious linearity of ${ }^{\wedge}$. The second identity is similarly proved, or it can be proved using the first identity as follows.

$$
B A B^{-1}=I+d \hat{d}, \quad d=a+\langle a, i b\rangle i b=a-\langle a, b\rangle b
$$

Remark 3.2. It holds true that $a \triangleright b=B^{-1} a$ for any $a, b \in \mathbb{C}^{2}$ with $T(B)=[b]$, since

$$
B^{-1} a=(I-b \hat{b}) a=a-b \hat{b} a=a-b\langle b, a\rangle=a+\langle a, b\rangle b=a \triangleright b .
$$

Also note that

$$
\begin{aligned}
\operatorname{tr}(A B) & =\operatorname{tr}((I+a \hat{a})(I+b \hat{b})) \\
& =\operatorname{tr}(I)+\operatorname{tr}(a \hat{a})+\operatorname{tr}(b \hat{b})+\operatorname{tr}(a \hat{a} b \hat{b}) \\
& =\operatorname{tr}(I)+0+0+\operatorname{tr}(\langle a, b\rangle a \hat{b}) \\
& =2-\langle a, b\rangle^{2}
\end{aligned}
$$

and hence $\langle a, b\rangle$ tells us about $\operatorname{tr}(A B)$. 


\section{PARABOlic REPRESEntations OF 2-BRidge KNOtS AND LinKS}

In this section, we investigate the set of parabolic representations of a 2-bridge link $K$ using its conway normal form $J\left(n_{1}, \cdots, n_{k}\right)=C\left[n_{1},-n_{2}, \cdots,(-1)^{k+1} n_{k}\right]$ and the symplectic quandle structure on $\mathcal{P}$ described in the previous section. Throughout the paper, we will write $K=J\left(n_{1}, \cdots, n_{k}\right)$ whenever we consider the link diagram $J\left(n_{1}, \cdots, n_{k}\right)$ for a link $K$.

Fix a Conway expansion $J\left(n_{1}, \cdots, n_{k}\right)$ of $K$ and its orientation. Then each parabolic representation corresponds to a $\mathfrak{C}$-coloring and vice versa. To get a $\mathfrak{C}$-coloring, we start from two vectors $a_{1,0}, b_{1,0}$ of $\mathbb{C}^{2}$ and obtain two $(i, j)$-vectors, $a_{i, j}, b_{i, j}$ for all $i=1, \cdots, k$ and

$$
j=0,1,2, \cdots, n_{i} \text { if } n_{i}>0 \text { and } j=0,-1,-2, \cdots, n_{i} \text { if } n_{i}<0,
$$

which are the $|j|$-th vectors of the $i$-th block in the diagram of $K$, consecutively obtained by performing the quandle operation of $\mathfrak{C}$ at every crossing while descending down. The last two vectors of each $i$-th block, $a_{i, n_{i}}, b_{i, n_{i}}$, will be denoted by $a_{i, f}, b_{i, f}$ sometimes for our convenience. (See Figure 4.)

At each crossing we will take "-" sign for a new vector, that is,

$$
a^{\prime}=b, \quad b^{\prime}=-(a \triangleright b)=-a-\langle a, b\rangle b
$$

when the crossing is given as in the left-hand side of Figure 2, and

$$
a^{\prime}=b, \quad b^{\prime}=-\left(a \triangleright^{-1} b\right)=-a+\langle a, b\rangle b
$$

when the crossing is given as in the right-hand side of Figure 2. Notice that our choice of
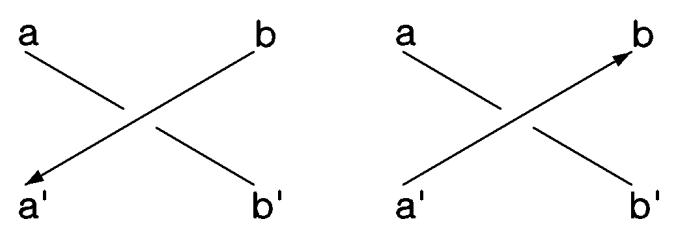

FiguRe 2.

"_" sign is to have a consistent determinant $\left\langle a^{\prime}, b^{\prime}\right\rangle=\langle a, b\rangle$ for each block and thus we get

$$
u_{i}=\left\langle a_{i, 0}, b_{i, 0}\right\rangle=\cdots=\left\langle a_{i, j}, b_{i, j}\right\rangle=\cdots=\left\langle a_{i, f}, b_{i, f}\right\rangle
$$

for each $i$, and $u_{i}$ will be called the determinant of $i$-th block.

If we let $\langle a, b\rangle=u$ and $X(u)=\left(\begin{array}{ll}0 & -1 \\ 1 & -u\end{array}\right)$, then Equation 4.1 and Equation 4.2 can be expressed as

$$
\left(a^{\prime}, b^{\prime}\right)=(a, b)\left(\begin{array}{cc}
0 & -1 \\
1 & -u
\end{array}\right)=(a, b) X(u) \quad \text { and } \quad\left(a^{\prime}, b^{\prime}\right)=(a, b)\left(\begin{array}{cc}
0 & -1 \\
1 & u
\end{array}\right)=(a, b) X(-u),
$$


respectively. Therefore we get from (4.3) that for each $i$

$$
\left(a_{i, j+\delta_{i}^{\prime}}, b_{i, j+\delta_{i}^{\prime}}\right)=\left(a_{i, j}, b_{i, j}\right) X\left(\delta_{i} u_{i}\right)^{\delta_{i}^{\prime}},
$$

where $\delta_{i}=1$ if the orientation of the crossing is downward and $\delta_{i}=-1$ if the orientation of the crossing is upward, and $\delta_{i}^{\prime}=1$ if $n_{i}>0$ and $\delta_{i}^{\prime}=-1$ if $n_{i}<0$ (see Figure 3).

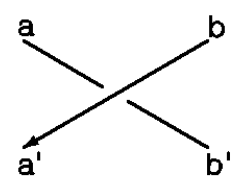

$\left(a^{\prime}, b^{\prime}\right)=(a, b) \times(u)$

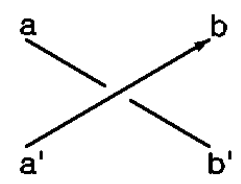

$\left(a^{\prime}, b^{\prime}\right)=(a, b) \times(-u)$

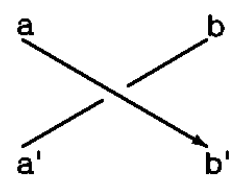

$\left(a^{\prime}, b^{\prime}\right)=(a, b) \times(u)^{-1}$

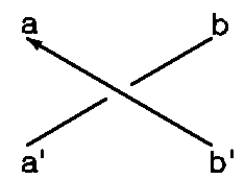

$\left(a^{\prime}, b^{\prime}\right)=(a, b) \times(-u)^{-1}$

FiguRE 3. quandle action when $u=\langle a, b\rangle$

Since the presentation of the knot group (or link group) of $K$ is generated by two meridians which are conjugate each other, and the meridians correspond to initial two vectors $a_{1,0}$ and $b_{1,0}$, there are 3 cases. The first case is that the representation is trivial, that is, in this case

$$
a_{1,0}=\left(\begin{array}{l}
0 \\
0
\end{array}\right)=b_{1,0}
$$

which corresponds to $\left(\begin{array}{ll}1 & 0 \\ 0 & 1\end{array}\right) \in S L(2, \mathbb{C})$. The second case is that the representation is a non-trivial abelian representation. In this case, we can normalize the meridians up to conjugate so that

$$
a_{1,0}=\left(\begin{array}{l}
1 \\
0
\end{array}\right), b_{1,0}=\left(\begin{array}{l}
v \\
0
\end{array}\right) \text {, }
$$

which corresponds respectively to $\left(\begin{array}{ll}1 & 1 \\ 0 & 1\end{array}\right)$ and $\left(\begin{array}{ll}1 & v^{2} \\ 0 & 1\end{array}\right)$ in $S L(2, \mathbb{C})$. The last case is that the representation is a non-abelian representation. In this case, we can normalize the meridians up to conjugate so that

$$
a_{1,0}=\left(\begin{array}{l}
1 \\
0
\end{array}\right), b_{1,0}=\left(\begin{array}{l}
0 \\
u
\end{array}\right), u \neq 0,
$$

which corresponds respectively to $\left(\begin{array}{ll}1 & 1 \\ 0 & 1\end{array}\right)$ and $\left(\begin{array}{cc}1 & 0 \\ -u^{2} & 1\end{array}\right)$ in $S L(2, \mathbb{C})$. Here $u^{2}=y$ in (2.3).

Note that the first and second cases are when $u=u_{1}=\left\langle a_{1,0}, b_{1,0}\right\rangle=0$ and the third case is when $u=u_{1}=\left\langle a_{1,0}, b_{1,0}\right\rangle \neq 0$. Note that the second case with $v \neq 1$ is possible only for links. Also, there is a parabolic representation $\rho: G(K) \rightarrow S L(2, \mathbb{C})$ with $\operatorname{tr}(\rho(a b))=2-u^{2}$

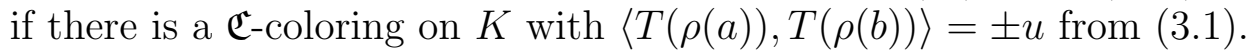



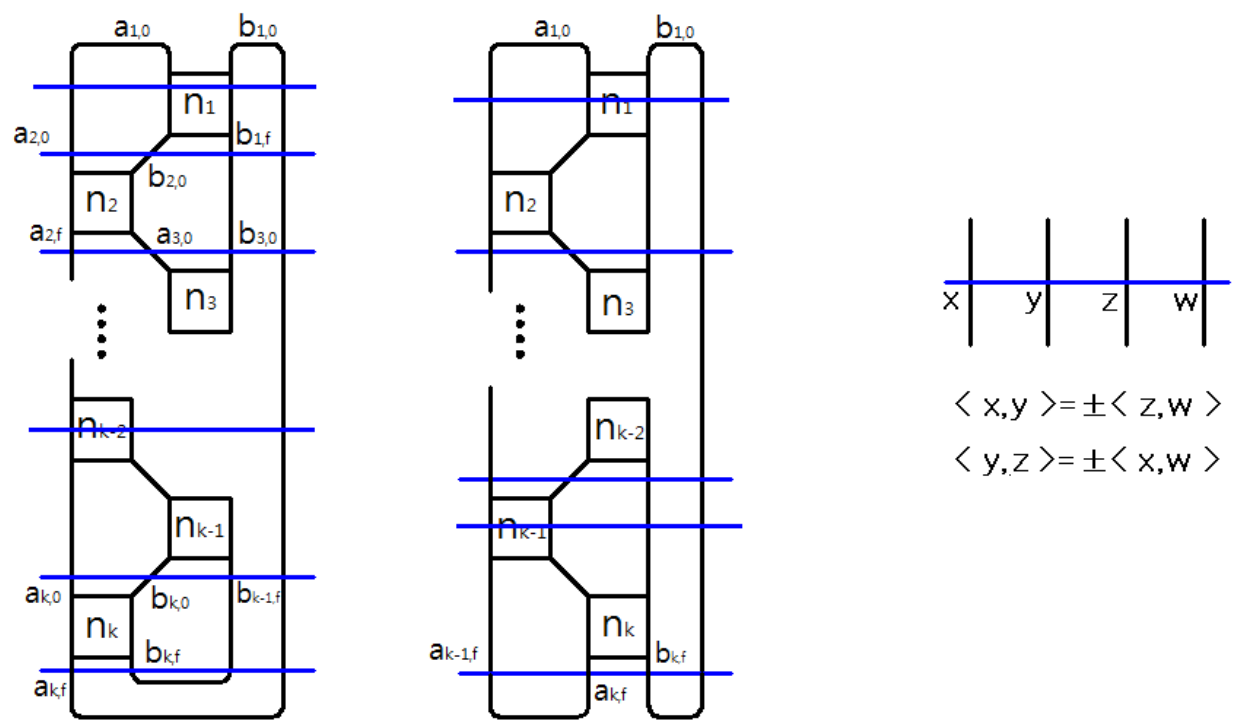

$\langle x, y\rangle= \pm\langle z, w\rangle$

$\langle y, z\rangle= \pm\langle x, w\rangle$

$$
a_{2,0}=a_{1,0}, a_{1, f}=b_{2,0}, b_{2, f}=a_{3,0}, \cdots
$$

FiguRE 4.

4.1. Key lemmas. In this section, we assume that the orientation of $K=J\left(n_{1}, \cdots, n_{k}\right)$ is given.

Lemma 4.1. Let $x, y, z, w$ are vectors in $\mathbb{C}^{2}$ which sequentially correspond to the 4 arc vectors intersecting an arbitrary horizontal line.(See Figure 4.) Then

$$
\langle x, y\rangle= \pm\langle z, w\rangle \quad \text { and } \quad\langle x, w\rangle= \pm\langle y, z\rangle .
$$

Proof. Since the element in $S L(2, \mathbb{C})$ corresponding to the loop rotating our diagram horizontally by 1 full turn is the identity matix, one of the following is satisfied:

(i) $X Y Z^{-1} W^{-1}=I d$

(ii) $X^{-1} Y^{-1} Z W=I d$

(iii) $X^{-1} Y Z W^{-1}=I d$

(iv) $X^{-1} Y Z^{-1} W=I d$

(v) $X Y^{-1} Z W^{-1}=I d$

(vi) $X Y^{-1} Z^{-1} W=I d$

where $X, Y, Z, W$ are the elements in $S L(2, \mathbb{C})$ which correspond to $x, y, z, w$, respectively. If (i) is satisfied, then

$$
\langle x, y\rangle^{2}=2-\operatorname{tr}(X Y)=2-\operatorname{tr}(W Z)=\langle z, w\rangle^{2}
$$

and

$$
\langle x, w\rangle^{2}=-\langle i x, w\rangle^{2}=-\left(2-\operatorname{tr}\left(X^{-1} W\right)\right)=-\left(2-\operatorname{tr}\left(Y Z^{-1}\right)\right)=-\langle y, i z\rangle^{2}=\langle y, z\rangle^{2} .
$$


In the case that any of (ii)-(vi) is satisfied, we also get the same result,

$$
\langle x, y\rangle^{2}=\langle z, w\rangle^{2}, \quad\langle x, w\rangle^{2}=\langle y, z\rangle^{2},
$$

by a similar argument. This completes the proof.

From now on, we will use $a$ and $b$ instead of $a_{1,0}$ and $b_{1,0}$ for simplicity if there is no worry about confusion.

Corollary 4.2. (i) $\left\langle b, a_{2 j, f}\right\rangle= \pm u_{2 j+1}$ if $2 j+1 \leq k$.

(ii) $\left\langle b, b_{2 j+1, f}\right\rangle= \pm u_{2 j+2}$ if $2 j+2 \leq k$.

(iii) $\left\langle a_{i, 0}, a_{i, f}\right\rangle= \pm\left\langle b_{i, 0}, b_{i, f}\right\rangle$ for each $i=1, \cdots, k$.

Proof. $\quad$ (i) By Lemma 4.1, $\left\langle b, a_{2 j, f}\right\rangle= \pm\left\langle a_{2 j+1,0}, b_{2 j+1,0}\right\rangle= \pm u_{2 j+1}$.

(ii) By Lemma 4.1. $\left\langle b, b_{2 j+1, f}\right\rangle= \pm\left\langle a_{2 j+2,0}, b_{2 j+2,0}\right\rangle= \pm u_{2 j+2}$.

(iii) The $\mathfrak{C}$-coloring of the $i$-th block of $J\left(n_{1}, \cdots, n_{k}\right)$ starting from two vectors $a_{1,0}, b_{1,0}$ with $u=u_{1}=\left\langle a_{1,0}, b_{1,0}\right\rangle$ is the same as the $\mathfrak{C}$-coloring of the first block of $K^{\prime}$, which is one of

$$
J\left(n_{i}\right), J\left(n_{i}, 2\right)
$$

with the same orientation as $K$ or reversed, starting from two vectors $a_{1,0}^{\prime}=a_{i, 0}$ and $b_{1,0}^{\prime}=b_{i, 0}$. If we apply Lemma 4.1 to $K^{\prime}$, then

$$
\left\langle a_{1,0}^{\prime}, a_{1, n_{i}}^{\prime}\right\rangle= \pm\left\langle b_{1,0}^{\prime}, b_{1, n_{i}}^{\prime}\right\rangle
$$

which implies

$$
\left\langle a_{i, 0}, a_{i, f}\right\rangle= \pm\left\langle b_{i, 0}, b_{i, f}\right\rangle .
$$

(iii) can be also proved directly by the same argument as Lemma 4.1 , because

$$
A_{i, 0}^{\epsilon_{1}} A_{i, f}^{\epsilon_{2}} B_{i, f}^{\epsilon_{3}} B_{i, 0}^{\epsilon_{4}}=I d
$$

for some $\epsilon_{1}, \epsilon_{2}, \epsilon_{3}, \epsilon_{4} \in\{1,-1\}$ with $\epsilon_{1}+\epsilon_{2}+\epsilon_{3}+\epsilon_{4}=0$.

The construction of $a_{i, j}$ and $b_{i, j}$ implies that there are polynomials $f_{i, j}(u), g_{i, j}(u), \tilde{f}_{i, j}(u), \tilde{g}_{i, j}(u)$ for each $i=1, \cdots, k$ and $j=0, \cdots, n_{i}$ such that

$$
a_{i, j}=f_{i, j}(u) a+g_{i, j}(u) b, \quad b_{i, j}=\tilde{f}_{i, j}(u) a+\tilde{g}_{i, j}(u) b,
$$

and these polynomials have the following properties.

Lemma 4.3. (i) $f_{i, j}(u), g_{i, j}(u), \tilde{f}_{i, j}(u), \tilde{g}_{i, j}(u)$ are monic polynomials with integer coefficients for any pair $(i, j)$ up to sign.

(ii) $u_{i}$ is also a monic integer coefficient polynomial of $u$ up to sign, and $u \mid u_{i}$ for all $i=1, \cdots, k$.

Proof. For $i=1$, (i) is obvious from the definition of $f_{i, j}(u), g_{i, j}(u), \tilde{f}_{i, j}(u), \tilde{g}_{i, j}(u)$ and (ii) is trivially satisfied because $u_{1}=u$. Since $u_{2}=\left\langle a_{2,0}, b_{2,0}\right\rangle=\left\langle a_{1,0}, a_{1, f}\right\rangle=u g_{1, f}(u)$, $u_{2}$ is a monic integer coefficient polynomial of $u$ and $u \mid u_{2}$ if $u_{2} \neq 0$, and obviously $f_{2, j}(u), g_{2, j}(u), \tilde{f}_{2, j}(u), \tilde{g}_{2, j}(u)$ are all monic polynomials with integer coefficients. Note thtat

$$
\operatorname{deg} f_{i, j+1}=\operatorname{deg} f_{i, j}+\operatorname{deg} u_{i}
$$


for any pair $(i, j)$, and the same is also true for $g_{i, j}(u), \tilde{f}_{i, j}(u), \tilde{g}_{i, j}(u)$.

Now we proceed by induction on $i$. Assume that the statement (i) and (ii) are true for all $i \leq k$. Since

and

$$
u_{k+1}= \pm\left\langle b, a_{k, f}\right\rangle= \pm u f_{k, f} \quad \text { when } k \text { is even }
$$

$$
u_{k+1}= \pm\left\langle b, b_{k, f}\right\rangle= \pm u \tilde{f}_{k, f} \quad \text { when } k \text { is odd }
$$

by Corollary 4.2, $u_{k+1}$ is also monic and is divided by $u$. Therefore we can conclude that (i) and (ii) are true for $i=k+1$, which completes the proof.

Lemma 4.4. Suppose $u=u_{1} \neq 0$. Let $x, y, z, w$ be vectors in $\mathbb{C}^{2}$ which sequentially correspond to the 4 arc vectors intersecting an arbitrary horizontal line (therefore $w=b_{1,0}$ ). Then the following holds for some $\epsilon \in\{1, i\}$.

(i) If $\langle x, y\rangle=0$ or $\langle z, w\rangle=0$, then $x= \pm \epsilon y$ and $z= \pm \epsilon w$.

(ii) If $\langle y, z\rangle=0$ or $\langle x, w\rangle=0$, then $y= \pm \epsilon z$ and $x= \pm \epsilon w$.

(iii) If $\langle x, z\rangle=0$, then $x= \pm \epsilon z$ and $w= \pm \epsilon\left(y \triangleright^{ \pm 1} z\right)$.

(iv) If $\langle y, w\rangle=0$, then $y= \pm \epsilon w$ and $z= \pm \epsilon\left(x \triangleright^{ \pm 1} y\right)$.

Here $\epsilon=i$ when the orientations are opposite, and the sign of $\triangleright^{ \pm 1}$ in (iii) (respectively, (iv)) is + only when the orientation of $z$ (respectively, $y$ ) is going down.

Proof. Note that by Lemma 4.1. $\langle x, y\rangle=0$ if and only $\langle z, w\rangle=0$, and $\langle x, w\rangle=0$ if and only $\langle y, z\rangle=0$.

Firstly, we prove the lemma for the case that the horizontal line cuts the first block, that is,

$$
x=a, y=a_{1, j}, z=b_{1, j}, w=b .
$$

Since an arc vector is multiplied by $i$ if its orientation is reversed, we may assume that the orientations of both of the two strands are going down as in Figure 5 . The arc vectors of the diagram in Figure 5 satisfy the following identity by Lemma 2.2 .

$$
\left(a_{1, k}, b_{1, k}\right)=(a, b) X(u)^{k}=(a, b)\left(\begin{array}{cc}
-p_{k-1}(-u) & -p_{k}(-u) \\
p_{k}(-u) & p_{k+1}(-u)
\end{array}\right) .
$$

(The left diagram is when $n_{1}>0$ and the right one is when $n_{1}<0$.)

If $\left\langle a, a_{1, j}\right\rangle=0$ then $p_{j}(-u)=0$ and so $p_{j-1}(-u)^{2}=p_{j+1}(-u)^{2}=1$ by (v) of Lemma 2.1. which implies

$$
a_{1, j}= \pm a, \quad b_{1, j}= \pm b .
$$

This proves that (i) is true for any $j$.

If $\left\langle a, b_{1, j}\right\rangle=0$, then $\left\langle a, a_{1, j+1}\right\rangle=0$ and thus

$$
b_{1, j}=a_{1, j+1}= \pm a, \quad a_{1, j} \triangleright b_{1, j}=b_{1, j+1}= \pm b
$$

holds true by (i), which proves (iii). If $\left\langle a_{1, j}, b\right\rangle=0$ then

$$
\left\langle b_{1, j-1}, b\right\rangle=0
$$

is satisfied and thus

$$
a_{1, j}=b_{1, j-1}= \pm b, \quad b_{1, j} \triangleright^{-1} a_{1, j}=a_{1, j-1}= \pm a
$$



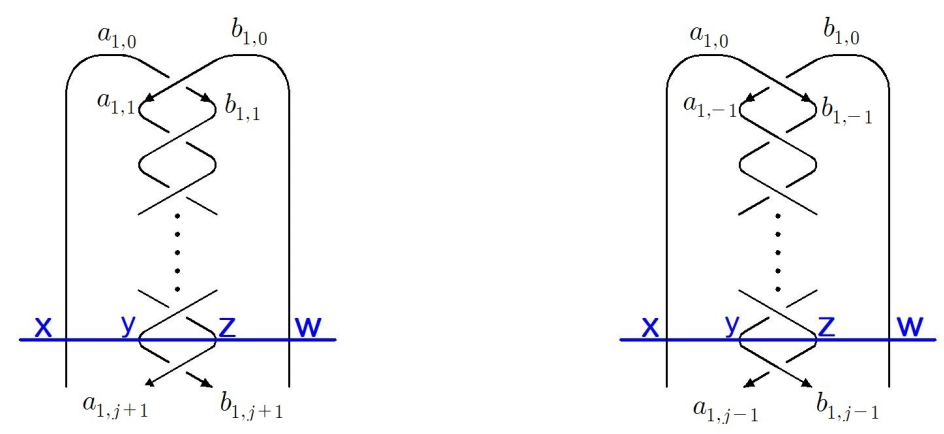

\section{FIGURE 5.}

holds true by (i), which proves (iv). Since $\langle y, z\rangle=\left\langle a_{1, j}, b_{1, j}\right\rangle=u \neq 0$, we have completed the proof for the case when the horizontal line cuts the first block.
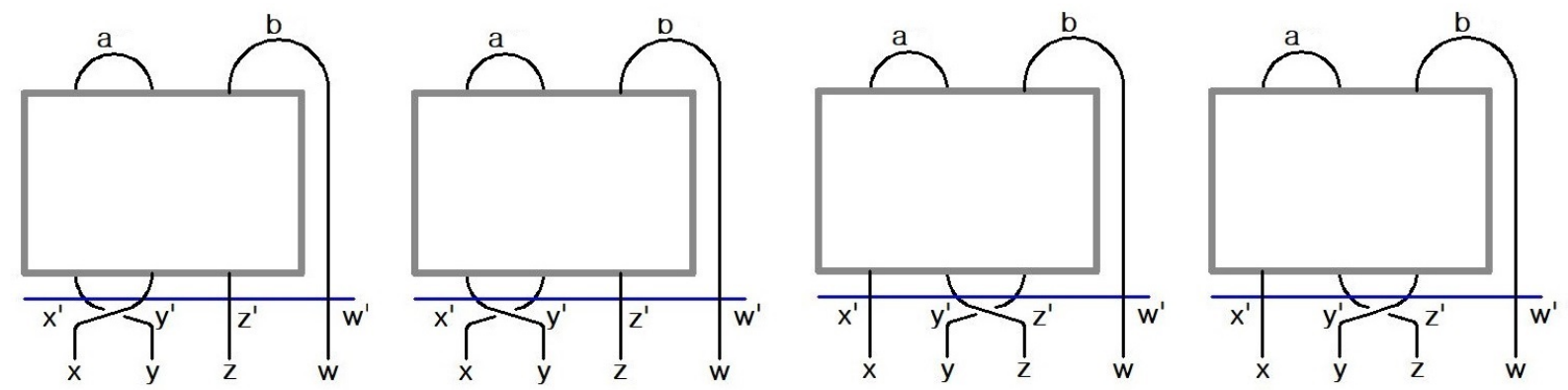

FiguRe 6.

Now we proceed by induction on $(i, j)$. Assume that the lemma is true for any four $(i, j)$ pairs such that $i<k$ or $i=k,|j|<m$ and consider the next four $(i, j)$ pairs of arc vectors $x, y, z, w$, that is to say $(i,|j|)=(k, m)$ if $m<\left|n_{k}\right|$ and $(i, j)=(k+1,0)$ if $m-1=\left|n_{k}\right|$. If we let $x^{\prime}, y^{\prime}, z^{\prime}, w^{\prime}$ be the 4 arc vectors on the previous horizontal line, then it can be proved that these four vectors satisfy one of the assumptions of (i), (ii), (iii), and (iv) if $x, y, z, w$ do. For example, in the case of $\langle x, y\rangle=0$, one of three equations,

$$
\left\langle x^{\prime}, y^{\prime}\right\rangle=0,\left\langle x^{\prime}, z^{\prime}\right\rangle=0,\left\langle y^{\prime}, w^{\prime}\right\rangle=0
$$

is satisfied by Lemma 4.1: $\left\langle x^{\prime}, y^{\prime}\right\rangle=0$ holds when $z^{\prime}=z$ and either $\left\langle x^{\prime}, z^{\prime}\right\rangle=0$ or $\left\langle y^{\prime}, w^{\prime}\right\rangle=0$ holds when $x=x^{\prime}$ (see Figure 6). Since the lemma is true for $x^{\prime}, y^{\prime}, z^{\prime}, w^{\prime}$ by the induction hypothesis, it is easy to show that $x= \pm \epsilon y$ and $z= \pm \epsilon w$, which means (i) is true for $x, y, z, w$. (ii), (iii), and (iv) are similarly proved.

Remark 4.5. If we let $x, y, z, w$ be vectors in Lemma 4.4 and $K^{\prime}$ be the knot or the link which is made by closing the 4 arcs of the upper tangle, as in the diagrams of Figure 7. 
Then the arc-coloring corresponds to a parabolic representation $\rho: G\left(K^{\prime}\right) \rightarrow S L(2, \mathbb{C})$ and the orientation of $K^{\prime}$ is the same as $K$ if $\epsilon=1$, and one of the two strand's orientations must be reversed if $\epsilon=i$.
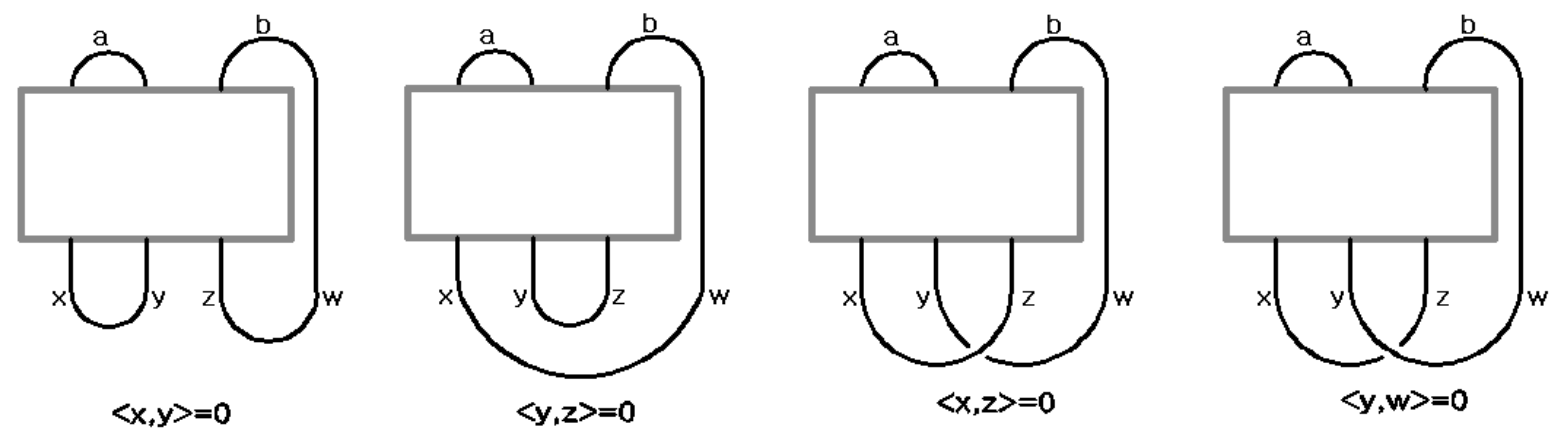

FiguRe 7.

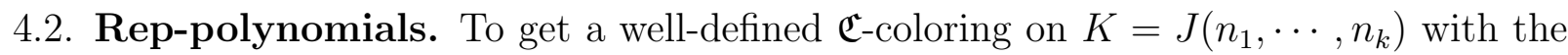
first two vectors $a=a_{1,0}$ and $b=b_{1,0}$, the last two vectors $a_{k, f}=a_{k, n_{k}}$ and $b_{k, f}=b_{k, n_{k}}$ must be the same, up to sign, as the vectors aleady determined for the arcs, which gives us the equation to determine the coloring (see Figure 8).
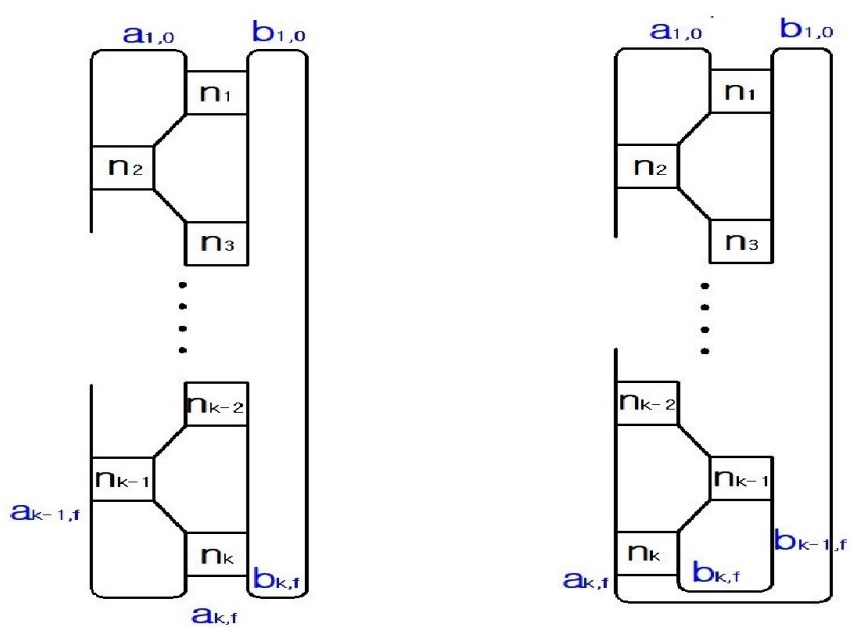

FiguRE 8 .

By Lemma 4.1.

$$
\left\langle a_{k, f}, a_{k-1, f}\right\rangle= \pm\left\langle b_{k, f}, b\right\rangle
$$

when $k$ is an odd number greater than 1 , and

$$
\left\langle b_{k, f}, b_{k-1, f}\right\rangle= \pm\left\langle a_{k, f}, b\right\rangle
$$


when $k$ is an even number. If we let $a_{0, f}=a$, then the above equation also holds for the case when $k=1$.

Let $P_{K}(u)$ be a polynomial in $u$ with positive leading coefficient which is defined as follows:

(i) $P_{K}(u)= \pm\left\langle a_{k, f}, a_{k-1, f}\right\rangle= \pm\left\langle b_{k, f}, b\right\rangle$ if $k$ is odd,

(ii) $P_{K}(u)= \pm\left\langle b_{k, f}, b_{k-1, f}\right\rangle= \pm\left\langle a_{k, f}, b\right\rangle$ if $k$ is even.

Note that $P_{K}(u)$ is defined for a diagram $K=J\left(n_{1}, \cdots, n_{k}\right)$, but we will see in Theorem 4.9 that it is essentially independent of the choice of a diagram.

Proposition 4.6. Let $K=J\left(n_{1}, \cdots, n_{k}\right)$ with an orientation. Then $P_{K}(u)$ is a monic polynomial with integer coefficients and there is a $\mathfrak{C}$-coloring with $\langle a, b\rangle=r$ on $K=$ $J\left(n_{1}, \cdots, n_{k}\right)$ if and only if $r$ is a root of the equation $P_{K}(u)=0$. Furthermore, 0 is a root of $P_{K}(u)$, which corresponds to an abelian representation, and there is a $\mathfrak{C}$-coloring with $\langle a, b\rangle=0, a \neq \pm b$ if and only if $K$ is a link.

Proof. We can choose an integer $n_{k+1}$ such that the orientation of $K$ is the same as $K^{\prime}=$ $J\left(n_{1}, \cdots, n_{k}, n_{k+1}\right)$. Then $P_{K}(u)$ equals $\pm u_{k+1}$ of $K^{\prime}$ by Corollary 4.2 and thus $P_{K}(u)$ is a monic polynomial with integer coefficients by Lemma 4.3 .

If we start with $a=b$, then $\left\{a_{i, j}, b_{i, j}\right\} \subset\{b,-b\}$ for all $i, j$, which implies $P_{K}(0)=0$ for any $K$. It is obvious that if $K$ is a link then any pair $a, b$ such that $\langle a, b\rangle=0$ always gives a $\mathfrak{C}$-coloring on $K$, but if $K$ is a knot then a $\mathfrak{C}$-coloring is obtained only when $a= \pm b$.

In the case of $\langle a, b\rangle \neq 0$, the followingh must be satisfied :

$$
\begin{aligned}
& \left\{\begin{array}{l}
a_{k, f}= \pm a_{k-1, f} \text { for odd } k \\
b_{k, f}= \pm b
\end{array}\right. \\
& \left\{\begin{array}{l}
a_{k, f}= \pm b \\
b_{k, f}= \pm b_{k-1, f}
\end{array} \text { for even } k\right.
\end{aligned}
$$

But by Lemma 4.4, (4.7) is equivalent to

$$
\left\langle a_{k, f}, a_{k-1, f}\right\rangle=0 \quad\left(\Leftrightarrow\left\langle b_{k, f}, b\right\rangle=0\right),
$$

and 4.8 is equivalent to

$$
\left\langle a_{k, f}, b\right\rangle=0 \quad\left(\Leftrightarrow\left\langle b_{k, f}, b_{k-1, f}\right\rangle=0\right) .
$$

Note that (i) $P_{K}(r)=0$ implies $P_{K}(-r)=0$, because if we get a $\mathfrak{C}$-coloring on $K$ from a pair $a, b$, then we must also get a $\mathfrak{C}$-coloring on $K$ from a pair $a,-b$ since it only changes the sign of the coloring from $a, b$, and (ii) each root $r$ of the equation $P_{K}(u)=0$ gives a parabolic representation of $K=J\left(n_{1}, \cdots, n_{k}\right)$ and there is no other parabolic representations. So we will call the polynomial $P_{K}(u)$, the rep-polynomial of a 2-bridge link $K$. Even though we defined the rep-polynomial when we have an orientation, but it does not depend on its orientation for the knot case and we have two rep-polynomials for the link case as we can see in the following Proposition. 
Proposition 4.7. Let $K=C\left[n_{1}, \cdots, n_{k}\right]$. If $-K$ and $\bar{K}$ are the orientation-reversed link of $K$ and the mirror of $K$ respectively, then

$$
P_{-K}(u)=P_{K}(u)=P_{\bar{K}}(u) .
$$

Especially, the rep-polynomial of $C\left[n_{1}, \cdots, n_{k}\right]$ equals the rep-polynomial of $C\left[-n_{1}, \cdots,-n_{k}\right]$.

Proof. Since there is a 1-1 correspondence between the set of $\mathfrak{C}$-colorings on $K$ and that of $-K$ by multiplying $i$ to each corresponding arc vector, and

$$
\langle i a, i b\rangle=-\langle a, b\rangle,
$$

$P_{-K}(u)=P_{K}(u)$ follows.

If we reflect an oriented diagram of $K=C\left[n_{1}, \cdots, n_{k}\right]$ in the mirror and then reverse its orientation, then we get an oriented diagram $\bar{K}$. It is easy to check that we get an well-defined $\mathfrak{C}$-coloring on $\bar{K}$ by reflecting any $\mathfrak{C}$-coloring on $K$. (See Figure 9.)
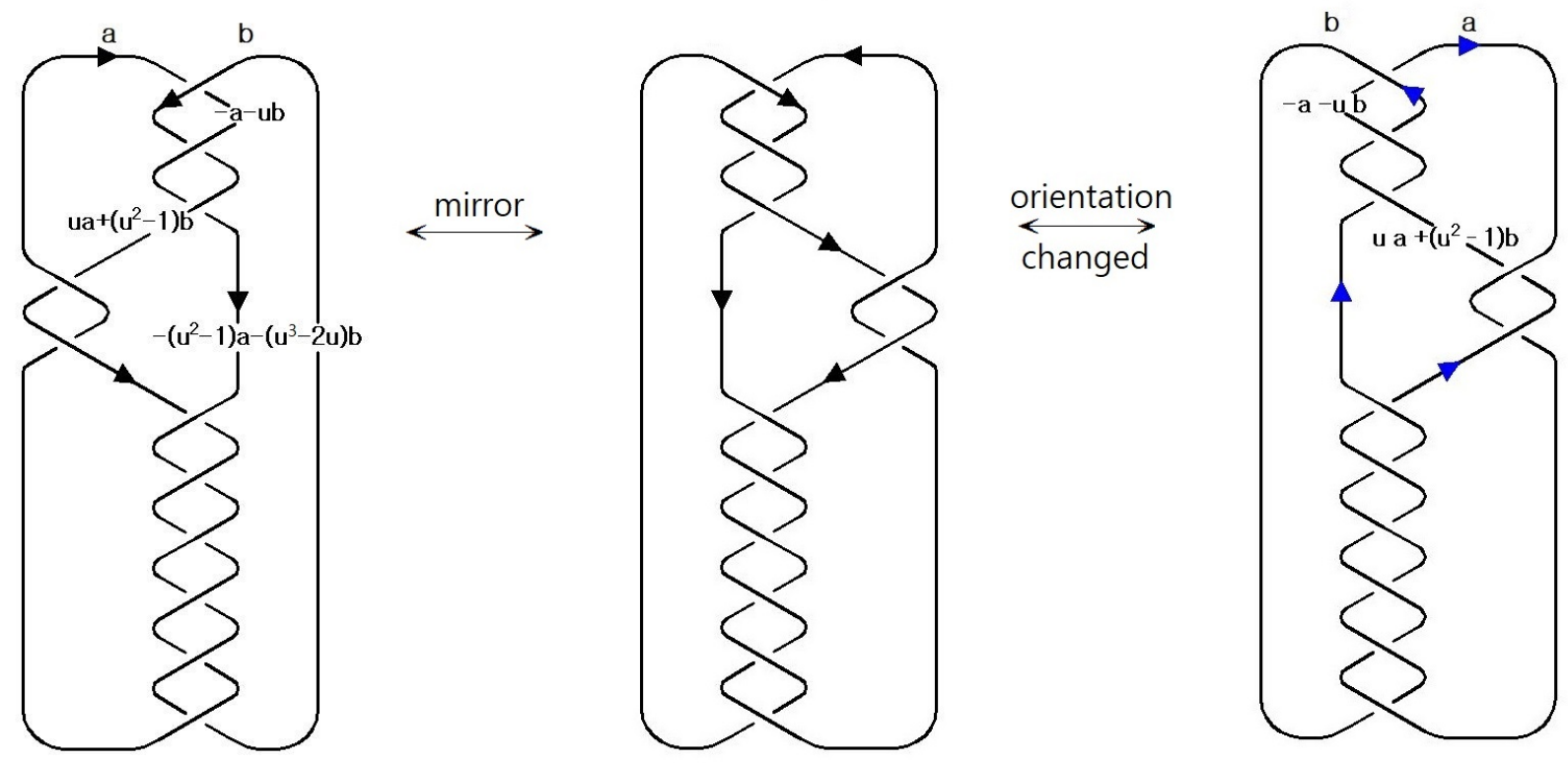

FiguRE 9.

Example 4.8. The last two vectors of the trefoil $K=3_{1}=C[3]$ are

$$
a_{1,3}=u a+\left(u^{2}-1\right) b, \quad b_{1,3}=-\left(u^{2}-1\right) a-\left(u^{3}-2 u\right) b,
$$

if we start with two vectors $a_{1,0}=a, b_{1,0}=b$ such that $u=\langle a, b\rangle$. (See Figure 10.) Therefore the rep-polynomial of $K$ is

$$
\left\langle b, b_{1,3}\right\rangle=u\left(u^{2}-1\right) .
$$




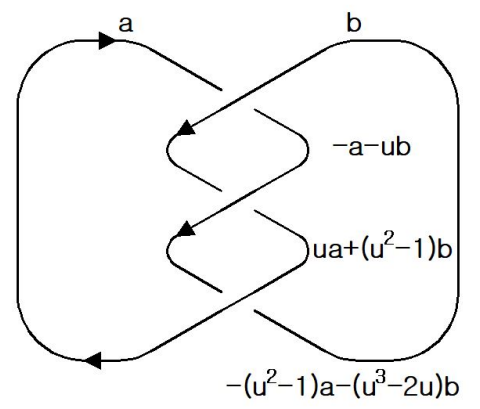

Figure 10. C[3]

By Proposition 4.7, we can define the rep-polynomial of $K$ without any specific orientation for a 2-bridge knot $K=C\left[n_{1}, \cdots, n_{k}\right]$. But if $K=C\left[n_{1}, \cdots, n_{k}\right]$ is a link, then we get a different rep-polynomial when we change the orientation of only one of the two components. So each link $K$ has two rep-polynomials, $P_{1}(u)$ and $P_{2}(u)$, up to its orientation, and these two satisfy

$$
P_{1}(i u)= \pm P_{2}(u), \quad P_{2}(i u)= \pm P_{1}(u)
$$

since

$$
\langle i a, b\rangle=i\langle a, b\rangle=\langle a, i b\rangle .
$$

Our definition of the rep-polynomial of a 2-bridge link depends on its diagram, but $P_{C\left[n_{1}, \cdots, n_{k}\right]}(u)=P_{C\left[m_{1}, \cdots, m_{l}\right]}(u)$ if $\left[n_{1}, \cdots, n_{k}\right]=\left[m_{1}, \cdots, m_{l}\right]$, since $C\left[n_{1}, \cdots, n_{k}\right]$ can be deformed into $C\left[m_{1}, \cdots, m_{l}\right]$ by a finite number of Reidemeister moves. Hence we have

Theorem 4.9. Let $K$ be a 2-bridge knot. Then there are only two rep-polynomials $P_{K}(u)$ and $P_{K}^{\prime}(u)$ for any Conway expansion diagram of $K$ and these two satisfy the followings.

(i) two diagrams $C\left[n_{1}, \cdots, n_{k}\right]$ and $C\left[m_{1}, \cdots, m_{k^{\prime}}\right]$ of $K$ have the same rep-polynomials if $\left[n_{1}, \cdots, n_{k}\right]= \pm\left[m_{1}, \cdots, m_{k^{\prime}}\right]$,

(ii) if $P_{K}(u)$ is the rep-polynomial of a diagram $C\left[n_{1}, \cdots, n_{k}\right]$, then $P_{K}^{\prime}(u)$ is the reppolynomial of the upside-down diagram $C\left[(-1)^{k+1} n_{k}, \cdots,(-1)^{k+1} n_{1}\right]$.

Similarly, each 2-bridge link $K$ has four rep-polynomials $P_{K}(u), P_{K}(i u), P_{K}^{\prime}(u), P_{K}^{\prime}(i u)$.

Each rational number $\frac{\beta}{\alpha} \in(0,1)$ corresponds to a 2-bridge link $C\left[n_{1}, \cdots, n_{k}\right]$ with $\left[n_{1}, \cdots, n_{k}\right]=\frac{\beta}{\alpha}$. So by Theorem 4.9 we have two polynomials

$$
P_{\frac{\beta}{\alpha}}(u), P_{\frac{\beta}{\alpha}}^{\prime}(u) \in \mathbb{Z}[u]
$$

if we give the downward-orientation on both components for the case when $C\left[n_{1}, \cdots, n_{k}\right]$ is a link. These polynomials satisfy the followings:

(i) $P_{\frac{\beta}{\alpha}}(u)=P_{C\left[n_{1}, \cdots, n_{k}\right]}(u)$ if $\left[n_{1}, \cdots, n_{k}\right]=\frac{\beta}{\alpha}$

(ii) $P_{\frac{\beta}{\alpha}}^{\alpha}(u)=P_{\frac{\beta^{\prime}}{\alpha^{\prime}}}(u)$ if and only if $\frac{\beta}{\alpha}=\frac{\beta^{\prime}}{\alpha^{\prime}}$ or $\frac{\alpha-\beta}{\alpha}=\frac{\beta^{\prime}}{\alpha^{\prime}}$ 
(iii) $P_{\frac{\beta^{\prime}}{\alpha^{\prime}}}(u)=P_{\frac{\beta}{\alpha}}^{\prime}(u)$, if $\frac{\beta}{\alpha}$ and $\frac{\beta^{\prime}}{\alpha^{\prime}}$ represent the same links and $\frac{\beta}{\alpha} \neq \frac{\beta^{\prime}}{\alpha^{\prime}}, \frac{\alpha-\beta}{\alpha} \neq \frac{\beta^{\prime}}{\alpha^{\prime}}$. In this case, $\alpha=\alpha^{\prime}, \beta \beta^{\prime} \equiv \pm 1(\bmod \alpha)$ if we assume that $(\alpha, \beta)=\left(\alpha^{\prime}, \beta^{\prime}\right)=1$.

Note that we will see later that if $(\alpha, \beta)=1$, then $\operatorname{deg} P_{\frac{\beta}{\alpha}}(u)=\alpha$ and

$$
\frac{1}{u^{\epsilon}} P_{\frac{\beta}{\alpha}}(u)= \pm \mathcal{R}\left(u^{2}\right)
$$

where $\mathcal{R}(y)$ is the Riley polynomial of $S(\alpha, \beta)$ and $\epsilon=1$ if $\alpha$ is odd, $\epsilon=2$ if $\alpha$ is even.

\section{3. $u_{i}$-sequence.}

Proposition 4.10. Let $K=C\left[n_{1}, \cdots, n_{k}\right]$ and $\alpha_{i}$ be an integer defined by

$$
\left[n_{1}, \cdots, n_{i}\right]=\frac{\beta_{i}}{\alpha_{i}}, \alpha_{0}=1
$$

Then

$$
\operatorname{deg}\left(u_{i}\right)=\alpha_{i-1}
$$

Proof. Using the induction on $i$ and the fact $\alpha_{i}=n_{i} \alpha_{i-1}+\alpha_{i-2}$, it is not difficult to show

$$
\begin{gathered}
\operatorname{deg}\left(u f_{2 j, f}(u)\right)=\alpha_{2 j}, \\
\operatorname{deg}\left(u f_{2 j+1, f}(u)\right)=\alpha_{2 j+1}-\alpha_{2 j}, \\
\operatorname{deg}\left(u \tilde{f}_{2 j+1, f}(u)\right)=\alpha_{2 j+1},
\end{gathered}
$$

and

$$
\operatorname{deg}\left(u \tilde{f}_{2 j, f}(u)\right)=\alpha_{2 j}-\alpha_{2 j-1} .
$$

Now by Corollary 4.2 we have

$$
u_{2 j+1}= \pm\left\langle b, a_{2 j, f}\right\rangle= \pm u f_{2 j, f}
$$

and

$$
u_{2 j+2}= \pm\left\langle b, b_{2 j+1, f}\right\rangle= \pm u \tilde{f}_{2 j+1, f},
$$

which completes the proof.

As we have seen in the proof of Proposition 4.6, $P_{K}(u)$ equals $u_{k+1}$ of either $C\left[n_{1}, \cdots, n_{k}, 1\right]$ or $C\left[n_{1}, \cdots, n_{k}, 2\right]$. Therefore we get the following corollary.

Corollary 4.11. Let $K=C\left[n_{1}, \cdots, n_{k}\right]$ and $\left[n_{1}, \cdots, n_{k}\right]=\frac{\beta}{\alpha}$. Then

(i) $u \mid P_{K}(u)$.

(ii) $\operatorname{deg} P_{K}(u)=\alpha$.

If $K=C\left[n_{1}, \cdots, n_{k}\right]$ and $C\left[n_{1}, \cdots, n_{m}\right], m>k$ have the same orientations on the arc corresponding to $a$ when we let the orientation of the arc corresponding to $b$ coincide, then the rep-polynomial $P_{K}(u)$ of $K$ and $u_{k+1}(u)$ of $C\left[n_{1}, \cdots, n_{m}\right]$ must be the same up to sign. If they have the opposite orientations on the arc corresponding to $a, P_{K}(u)= \pm i^{\alpha} u_{k+1}(i u)$ when $\left[n_{1}, \cdots, n_{k}\right]=\frac{\beta}{\alpha}$. So we have the following. 
Corollary 4.12. Let $K=C\left[n_{1}, \cdots, n_{k}\right]$ and $\left[n_{1}, \cdots, n_{j}\right]=\frac{\beta_{j}}{\alpha_{j}},\left(\alpha_{j}, \beta_{j}\right)=1$. Then $u_{j+1}$ is either $P_{\frac{\beta_{j}}{\alpha_{j}}}(u)$ or $\pm i^{\alpha_{j}} P_{\frac{\beta_{j}}{\alpha_{j}}}(i u)$.

Definition 4.13. The $u_{i}$-sequence of $K=C\left[n_{1}, \cdots, n_{k}\right]$ is defined as the sequence of polynomials in $\mathbb{Z}[u]$,

$$
\left(u_{1}, u_{2}, \cdots, u_{k}\right)
$$

where $u_{1}(u)=u$. We will call the sequence of numbers,

$$
\left(r, u_{2}(r), \cdots, u_{k}(r)\right)
$$

for a non-zero root of the rep-polynomial of $K$, the $u_{i}(r)$-sequence of $K$.

We can observe that the $u_{i}$-sequence of $K=C\left[n_{1}, \cdots, n_{k}\right]$ is

$$
\left(u, \pm \epsilon_{1}^{\alpha_{1}} P_{\frac{\beta_{1}}{\alpha_{1}}}\left(\epsilon_{1} u\right), \pm \epsilon_{2}^{\alpha_{2}} P_{\frac{\beta_{2}}{\alpha_{2}}}\left(\epsilon_{2} u\right), \cdots, \pm \epsilon_{k-1}^{\alpha_{k-1}} P_{\frac{\beta_{k-1}}{\alpha_{k-1}}}\left(\epsilon_{k-1} u\right)\right),
$$

where $\epsilon_{j} \in\{1, i\}$ for each $j=1,2, \cdots, k-1$.

Remark 4.14. For each root $r$ of $P_{K}(u)$ and for each $i, u_{i}(r)$ is related to the trace of the element $A_{i}$ in $S L(2, \mathbb{C})$ corresponding to the loop rotating the $i$-th block horizontally by 1 full turn. More precisely, the trace of $A_{i}$ is equal to $2-u_{i}(r)^{2}$,

$$
\operatorname{tr} A_{i}=2-u_{i}(r)^{2} \text {. }
$$

The following is an immediate consequence of the definitions for $u_{i}$ and $P_{K}(u)$.

Proposition 4.15. Let $K$ be $C\left[n_{1}, n_{2}, \cdots, n_{k}\right]$ and $K^{\prime}$ be its upside-down. Suppose $P_{K}(u)$ and $P_{K^{\prime}}(u)$ are their rep-polynomials and $\left(u_{1}, u_{2}, \cdots, u_{k}\right)$ and $\left(u_{1}^{\prime}, u_{2}^{\prime}, \cdots, u_{k}^{\prime}\right)$ are their $u_{i}$-sequences. Then $P_{K^{\prime}}(u)=P_{K}^{\prime}(u)$ and satisfies the following.

(i) If $P_{K}(r)=0$ then $P_{K}^{\prime}\left(u_{k}(r)\right)=0$ and $u_{k}^{\prime}\left(u_{k}(r)\right)^{2}=r^{2}$.

(ii) If $P_{K}^{\prime}(s)=0$ then $P_{K}\left(u_{k}^{\prime}(s)\right)=0$ and $u_{k}\left(u_{k}^{\prime}(s)\right)^{2}=s^{2}$.

Lemma 4.16. Let $K$ be $C\left[n_{1}, n_{2}, \cdots, n_{m}\right]$ with a fixed orientation and $-K$ be the orientationreversed diagram of $K$. Let $P_{i}(u)$ and $\tilde{P}_{i}(u)$ be the $u_{i}$ of $K$ and $-K$. Then for each $i=1, \cdots, m$,

$$
\tilde{P}_{i}(u)=-P_{i}(-u)= \pm P_{i}(u)
$$

Proof. If $\left\{a_{i, j}, b_{i, j}\right\}$ is a $\mathfrak{C}$-coloring on $K$, then $\left\{i a_{i, j}, i b_{i, j}\right\}$ is a $\mathfrak{C}$-coloring on $\tilde{K}$. Hence

$$
\tilde{P}_{i}(-u)=\left\langle i a_{i, j}, i b_{i, j}\right\rangle=-\left\langle a_{i, j}, b_{i, j}\right\rangle=-P_{i}(u) .
$$

Since $P_{i}(u)$ is an even polynomial or an odd polynomial by Corollary 4.25 , we get

$$
\tilde{P}_{i}(u)=-P_{i}(-u)= \pm P_{i}(u) \text {. }
$$

Proposition 4.17. Let $K=C\left[n_{1}, n_{2}, \cdots, n_{m}\right]$ with a fixed orientation and $K^{\prime}$ be the upside-down of $K$. Suppose that $r$ is a non-zero root of the rep-polynomial of $K$. Then $\left(u_{m}(r), \cdots, u_{2}(r), r\right)$ is the $u_{i}\left(u_{m}(r)\right)$-sequence of $K^{\prime}$ up to sign. 
Proof. Suppose that $\left\{a_{i, j}, b_{i, j} \mid i=1, \cdots, m, j=0, \cdots, n_{i}\right\}$ is a $\mathfrak{C}$-coloring on $K$ such that $\left\langle a_{1,0}, b_{1,0}\right\rangle=r$, and $\left\{A_{i, j}, B_{i, j}\right\}$ are the elements in $S L(2, \mathbb{C})$ which correspond to the representation. Then the outermost coloring vectors are unchanged by the half rotation up to sign, that is, $\left[a_{1,0}\right],\left[b_{1,0}\right],\left[a_{2 i, j}\right],\left[a_{m, f}\right],\left[b_{m, f}\right]$ and $\left[a_{2 i, f}\right]$ are all unchanged for any $2 i \leq m$ and $j=0, \cdots, n_{2 i}$. This implies that each $u_{2 i+1}=\left\langle a_{2 i, f}, b_{1,0}\right\rangle$ is also unchanged by the half rotation up to sign.

For any $i$ such that $2 i \leq m$,

$$
A_{2 i, 0} B_{2 i, 0}=\cdots=A_{2 i, j} B_{2 i, j}=\cdots=A_{2 i, n_{2 i}} B_{2 i, n_{2 i}}
$$

and $A_{2 i, j} B_{2 i, j}$ is not changed by the half rotation. Hence the half rotations preserve each $u_{2 i}^{2}=2-\operatorname{tr}\left(A_{2 i, j} B_{2 i, j}\right)$.

4.4. Non-abelian representations. We have seen in the previous section that the degree of the rep-polynomial $P_{K}(u)$ of $K=C\left[n_{1}, \cdots, n_{k}\right]=S(\alpha, \beta)$ is $\alpha$ and $u$ is a factor of $P_{K}(u)$. Therefore if $K$ is a knot, then $u^{m} \nmid P_{K}(u)$ for any $m>1$ and all the roots of $\frac{1}{u} P_{K}(u)$ give non-abelian representations of $K$, since the degree of the Riley Polynomial $\mathcal{R}(y)$ of $K$ is $\frac{\alpha-1}{2}$ and $y=u^{2}$. We give a direct proof for this here:

Lemma 4.18. Let $P_{K}(u)$ be the rep-polynomial of $K=C\left[n_{1}, \cdots, n_{k}\right]$. Then $u^{2} \mid P_{K}(u)$ if and only if $K$ is a link. Furthermore, if $K$ is a knot then $\frac{1}{u} P_{K}(u) \in \mathbb{Z}[u]$ is a monic polynomial whose constant term is either 1 or -1 .

Proof. Note that

and

$$
P_{K}(u)= \pm u f_{k, f}(u) \quad \text { when } \quad k \quad \text { is even }
$$

$$
P_{K}(u)= \pm u \tilde{f}_{k, f}(u) \quad \text { when } \quad k \quad \text { is odd. }
$$

Now we assume that $\langle a, b\rangle=0$. Then $\left\{a_{i, j}, b_{i, j}\right\} \subset\{a,-a, b,-b\}$ for all $i, j$. If $K$ is a link, then

which implies

$$
a_{k, f}= \pm b(k: \text { even }) \quad \text { or } \quad b_{k, f}= \pm b(k: \text { odd }),
$$

or

$$
f_{k, f}(0)=0 \quad \text { and } \quad g_{k, f}(0)= \pm 1 \quad(k: \text { even })
$$

$$
\tilde{f}_{k, f}(0)=0 \quad \text { and } \quad \tilde{g}_{k, f}(0)= \pm 1 \quad(k: \text { odd }) .
$$

(See Figure 11.) This proves that if $K$ is a link then $\left.\frac{1}{u} P_{K}(u)\right|_{u=0}=0$ and thus $u^{2}$ divides $P_{K}(u)$.

If $K$ is a knot, then

$$
a_{k, f}= \pm a(k: \text { even }) \quad \text { or } \quad b_{k, f}= \pm a(k: \text { odd }),
$$

which implies that

$$
f_{k, f}(0)= \pm 1 \neq 0(k: \text { even }) \quad \text { or } \quad \tilde{f}_{k, f}(0)= \pm 1 \neq 0(k: \text { odd })
$$

and thus there is $c_{2 k} \in \mathbb{Z}, k=1,2, \cdots, \frac{\alpha-3}{2}$ such that

$$
P_{K}(u)=u\left(u^{\alpha-1}+c_{\alpha-3} u^{\alpha-3}+\cdots+c_{2} u^{2} \pm 1\right) .
$$



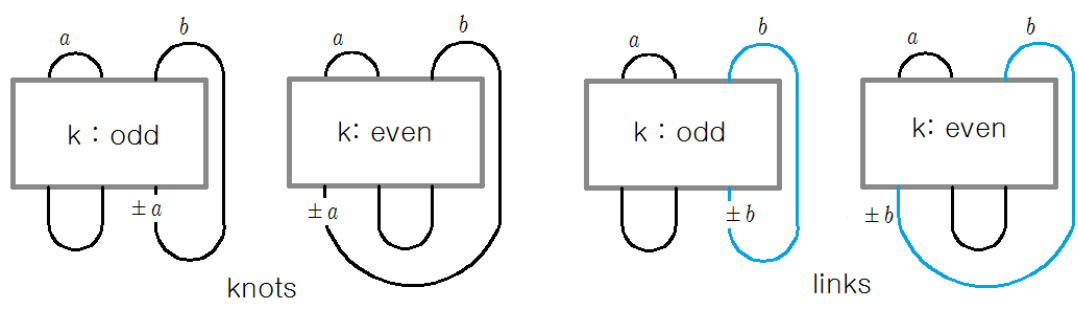

FiguRE 11. $u=0$

This proves

$$
\left.\frac{1}{u} P_{K}(u)\right|_{u=0} \neq 0
$$

and the last statement.

Theorem 4.19. Let $P_{K}(u)$ be the rep-polynomial of a knot $K=C\left[n_{1}, \cdots, n_{k}\right]$. Then there is a 1-1 correspondence between the set of the squares of the roots of $\frac{1}{u} P_{K}(u)$ and the set of the non-abelian representations of $K$.

Proof. By Lemma 4.18, all the roots of $\frac{1}{u} P_{K}(u)$ are nonzero. Since each root of $P_{K}(u)$ corresponds to a $\mathfrak{C}$-coloring on $K$ by Proposition 4.6, all the roots of $\frac{1}{u} P_{K}(u)$ give nonabelian representations of $K$. This correspondence is bijective, because the degree of $\frac{1}{u} P_{K}(u)$ is equal to that of $\mathcal{R}\left(u^{2}\right)$, and $\mathcal{R}(y)$ has no repeated roots.

Remark 4.20. If we take any Conway's normal form $K=C\left[n_{1}, \cdots, n_{k}\right]$ of a 2-bridge knot $K=S(\alpha, \beta)$ such that

$$
\left[n_{1}, n_{2}, \cdots, n_{k}\right]= \pm \frac{\beta}{\alpha} \text { or } \pm \frac{\alpha-\beta}{\alpha}
$$

then we can obtain the Riley polynomial of $K$ from our polynomial $\frac{1}{u} P_{K}(u)$ by converting $u^{2}$ into $y$. That is,

$$
\frac{1}{u} P_{\frac{\beta}{\alpha}}(u)=\frac{1}{u} P_{\frac{\alpha-\beta}{\alpha}}(u)= \pm \mathcal{R}\left(u^{2}\right)= \pm W_{11}\left(u^{2}\right) .
$$

Note that the relation $W \rho(a) W^{-1}=\rho(w) \rho(a) \rho(w)^{-1}=\rho(b)$ with 2.1 and 2.3 implies that

and

$$
\left[\begin{array}{l}
W_{11}\left(u^{2}\right) \\
W_{21}\left(u^{2}\right)
\end{array}\right]=T\left(W \rho(a) W^{-1}\right)=T(\rho(b))=\left[\begin{array}{l}
0 \\
u
\end{array}\right]
$$

$$
\left\langle\left(\begin{array}{l}
W_{11}\left(u^{2}\right) \\
W_{21}\left(u^{2}\right)
\end{array}\right),\left(\begin{array}{l}
0 \\
u
\end{array}\right)\right\rangle=u W_{11}\left(u^{2}\right)= \pm P_{\frac{\beta}{\alpha}}(u) .
$$

For example, $K_{1}=S(7,3)$ and $K_{2}=S(7,5)$ are equivalent 2-bridge knots and their corresponding Conway's normal forms are $K_{1}=C[2,3]$ and $K_{2}=C[1,2,2]$, which is the upside -down of $C[2,3]$. The rep-polynomials of $C[2,3]$ and $C[1,2,2]$ are $u\left(u^{6}-u^{4}+2 u^{2}-1\right)$ 
and $u\left(u^{6}+3 u^{4}+2 u^{2}-1\right)$, respectively. The Riley polynomials of $K_{1}$ and $K_{2}$ are $-\left(y^{3}-\right.$ $\left.y^{2}+2 y-1\right)$ and $-\left(y^{3}+3 y^{2}+2 y-1\right)$, respectively.

All the rep-polynomials of 2-bridge knots are expressed as combinations of Chebyshev polynomials $p_{j}$ 's. (See Appendix.) So we can also get an explicit formula for Riley polynomial.

Remark 4.21. For a link $K$, we have

$$
P_{K}(u)= \pm u^{2} \mathcal{R}\left(u^{2}\right)= \pm u^{2} W_{12}\left(u^{2}\right)= \pm W_{21}^{*}\left(u^{2}\right)
$$

since the degree of both $P_{K}(u)$ and $\pm u^{2} \mathcal{R}\left(u^{2}\right)$ is $\alpha$. Hence there is a 1-1 correspondence between the set of the squares of the non-zero roots of $\frac{1}{u^{2}} P_{K}(u)$ and the set of the nonabelian representations of $K$. But we will see that $\frac{1}{u^{2}} P_{K}(u)$ might have 0 as a root later.

Note that the relation $W^{*} \rho(a)\left(W^{*}\right)^{-1}=\rho\left(w^{*}\right) \rho(a) \rho\left(w^{*}\right)^{-1}=\rho(a)$ with 2.3) and 2.5) implies

$$
\left[\begin{array}{l}
W_{11}^{*}\left(u^{2}\right) \\
W_{21}^{*}\left(u^{2}\right)
\end{array}\right]=T\left(W^{*} \rho(a)\left(W^{*}\right)^{-1}\right)=T(\rho(a))=\left[\begin{array}{l}
1 \\
0
\end{array}\right],
$$

and the relation $W \rho(b) W^{-1}=\rho(w) \rho(b) \rho(w)^{-1}=\rho(b)$ with (2.3) and 2.5) implies that

$$
\begin{aligned}
\left.\left(\left(W \rho(b) W^{-1}\right)\right)^{-1}\right) & =T\left(W\left(\begin{array}{cc}
0 & -\frac{1}{u} \\
u & 0
\end{array}\right) \rho(a)\left(\begin{array}{cc}
0 & -\frac{1}{u} \\
u & 0
\end{array}\right)^{-1} W^{-1}\right) \\
& =T\left(W\left(\begin{array}{cc}
0 & -\frac{1}{u} \\
u & 0
\end{array}\right) \rho(a)\left(W\left(\begin{array}{cc}
0 & -\frac{1}{u} \\
u & 0
\end{array}\right)\right)^{-1}\right) \\
& =\left[\begin{array}{l}
u W_{12}\left(u^{2}\right) \\
u W_{22}\left(u^{2}\right)
\end{array}\right]=\left[\begin{array}{l}
0 \\
u
\end{array}\right]=T(\rho(b)) .
\end{aligned}
$$

And we can observe

$$
\left\langle\left(\begin{array}{l}
W_{11}^{*}\left(u^{2}\right) \\
W_{21}^{*}\left(u^{2}\right)
\end{array}\right),\left(\begin{array}{l}
1 \\
0
\end{array}\right)\right\rangle=-W_{21}^{*}\left(u^{2}\right)=u^{2} W_{12}\left(u^{2}\right)=\left\langle\left(\begin{array}{l}
u W_{12}\left(u^{2}\right) \\
u W_{22}\left(u^{2}\right)
\end{array}\right),\left(\begin{array}{l}
0 \\
u
\end{array}\right)\right\rangle .
$$

Remark 4.22. The last statement of Lemma 4.18 implies that the Riley polynomial $(-1)^{\frac{\alpha-1}{2}} \mathcal{R}(y) \in \mathbb{Z}[y]$ is a monic polynomial whose constant term is either 1 or -1 . (Note that (4.12) is equivalent to Equation 3.10 of [32.) But this does not hold for 2-bridge links. For example, the rep-polynomial of $C[2,1,2]$, the Whitehead link, is $u^{4}\left(u^{4} \pm 2 u^{2}+2\right)$.

Example 4.23. We have seen in Example 4.8 that the rep-polynomial of $C[3]$ is

$$
\left\langle b, b_{1,3}\right\rangle=u\left(u^{2}-1\right) .
$$

Therefore the trefoil has only one non-abelian parabolic representation because 1 and -1 correspond to the same $\mathfrak{C}$-coloring on $K$. This representation has a generating meridian pair $\left(\begin{array}{ll}1 & 1 \\ 0 & 1\end{array}\right),\left(\begin{array}{cc}1 & 0 \\ -1 & 1\end{array}\right)$ up to conjugation.

Example 4.24. The rep-polynomial of a knot $K=C[2,2,5]$ is

$$
P_{K}(u)=u(u-1)(u+1) h(u) h(-u)
$$


where

$$
h(u)=u^{12}-2 u^{10}+u^{9}+4 u^{8}-u^{7}-3 u^{6}+3 u^{5}+3 u^{4}-u^{3}-u^{2}+2 u+1
$$

and all the non-zero roots of $P_{K}(u)$ give non-abelian representations.

We can easily check that the $u_{i}$-sequence of $K=C[2,2,5]$ is

$$
\left(u,-u^{2},-u\left(u^{4}-u^{2}+1\right)\right)=\left(u,-P_{C[2]}(u),-i P_{C[2,2]}(i u)\right) .
$$

Corollary 4.25. For any $K=C\left[n_{1}, \cdots, n_{k}\right], u_{i} \in \mathbb{Z}[u]$ is either an odd polynomial or an even polynomial depending on whether $K$ is a knot or a link respectively.

Proof. It follows from 4.13), (4.14), and Corollary 4.12 that if $C\left[n_{1}, \cdots, n_{i-1}\right]$ is a knot, then $u_{i}$ is an odd polynomial in $u$ and if $C\left[n_{1}, \cdots, n_{i-1}\right]$ is a link, then $u_{i}$ is an even polynomial.

\section{TRACE FIELD}

The trace field of a representation $\rho$ is defined by $\mathbb{Q}\langle\operatorname{tr} \rho(\gamma) \mid \gamma \in G(K)\rangle$ [26]. So it is obviously equal to $\mathbb{Q}(y)=\mathbb{Q}\left(u^{2}\right)$ by $(2.3)$. In this section, we show that any rep-polynomial of a 2-bridge knot always has a special decomposition, and $u \in \mathbb{Q}\left(u^{2}\right)$ as a unit.

Theorem 5.1. Let $K$ be a 2-bridge knot. Then the rep-polynomial of $K$ is

$$
P_{K}(u)=u g(u) \hat{g}(u)
$$

for some $g(u) \in \mathbb{Z}[u]$ such that $\hat{g}(u)=(-1)^{\operatorname{deg} g} g(-u)$ and $\hat{g}(u) \neq g(u)$. Furtheremore there is a 1-1 correspondence between the set of roots of $g(u)$ and the set of non-abelian parabolic representations of $K$.

Proof. Every 2-bridge knot $K$ can be expressed as

$$
K=C\left[2 n_{1}, 2 n_{2}, \cdots, 2 n_{2 m}\right], n_{i} \in \mathbb{Z}-\{0\},
$$

so called a reduced even expansion of $K$. (See [11] or [12].) In such a diagram for $K$,

(i) $u_{2 k}= \pm P_{C\left[2 n_{1}, 2 n_{2}, \cdots, 2 n_{2 k-1}\right]}(u)$ is an even polynomial in $u$,

(ii) $u_{2 k+1}= \pm P_{C\left[2 n_{1}, 2 n_{2}, \cdots, 2 n_{2 k}\right]}(u)$ is an odd polynomial in $u$,

as one can easily see that $C\left[2 n_{1}, 2 n_{2}, \cdots, 2 n_{2 k}\right]$ is a knot and $C\left[2 n_{1}, 2 b_{2}, \cdots, 2 n_{2 k-1}\right]$ is a link for any $k$ such that $2 k+1<2 m$. For example, $u_{2}=-u^{2} p_{n_{1}}\left(-2-u^{2}\right)$. (See Appendix B for the details.)

So $u_{2 m}=f\left(u^{2}\right)$ for some $f \in \mathbb{Z}[u]$. Now if we let $\pm r_{1}, \cdots, \pm r_{k}$ be the non-zero roots of the rep-polynomial $P_{K}(u)$, then the non-zero roots of the rep-polynomial $P_{K}^{\prime}(u)$ which comes from the upside-down diagram, or equivalently the rep-polynomial coming from the arc coloring which starts with the two vectors on the bottom, are

$$
\pm f\left(r_{1}^{2}\right), \cdots, \pm f\left(r_{k}^{2}\right)
$$

By the fact that all the roots of the Riley polynomial are distinct [32], $r_{i} \neq r_{j}$ for $i \neq j$, and thus

$$
P_{K}(u)=u\left(u+r_{1}\right)\left(u-r_{1}\right) \cdots\left(u+r_{k}\right)\left(u-r_{k}\right) .
$$


and

Since

$$
P_{K}^{\prime}(u)=u\left(u+f\left(r_{1}^{2}\right)\right)\left(u-f\left(r_{1}^{2}\right)\right) \cdots\left(u+f\left(r_{k}^{2}\right)\right)\left(u-f\left(r_{k}^{2}\right)\right)
$$

$$
\begin{gathered}
P_{K}(u)=u\left(u+r_{1}\right)\left(u-r_{1}\right) \cdots\left(u+r_{k}\right)\left(u-r_{k}\right)=u\left(u^{2}-r_{1}^{2}\right) \cdots\left(u^{2}-r_{k}^{2}\right) \in \mathbb{Z}[u], \\
\left(u-r_{1}^{2}\right) \cdots\left(u-r_{k}^{2}\right) \in \mathbb{Z}[u] .
\end{gathered}
$$

As elementary symmetric polynomials of $f\left(a_{1}\right), f\left(a_{2}\right), \cdots, f\left(a_{k}\right)$ can be expressed as those of $a_{1}, a_{2}, \cdots, a_{k}$,

$$
\begin{gathered}
g^{\prime}(u):=\left(u-f\left(r_{1}^{2}\right)\right) \cdots\left(u-f\left(r_{k}^{2}\right)\right) \in \mathbb{Z}[u], \\
P_{K}^{\prime}(u)=u g^{\prime}(u) \hat{g}^{\prime}(u) .
\end{gathered}
$$

Similarly, since $P_{K}=\left(P^{\prime}\right)_{K}^{\prime}$ there is a polynomial $h$ with integer coefficients such that

$$
P_{K}(u)=u\left(u+h\left(f\left(r_{1}^{2}\right)\right)\right)\left(u-h\left(f\left(r_{1}^{2}\right)\right)\right) \cdots\left(u+h\left(f\left(r_{k}^{2}\right)\right)\right)\left(u-h\left(f\left(r_{k}^{2}\right)\right)\right)
$$

and $g(u):=\left(u-h\left(f\left(r_{1}^{2}\right)\right)\right) \cdots\left(u-h\left(f\left(r_{k}^{2}\right)\right)\right) \in \mathbb{Z}[u]$, and

$$
P_{K}(u)=u g(u) \hat{g}(u)
$$

as desired. The property $\hat{g}(u) \neq g(u)$ is obvious, because if it is not the case then $P_{K}(u)=$ $u g(u)^{2}$ and this contradicts that all the roots of the Riley polynomial are distinct.

Since two $\mathfrak{C}$-colorings on $K$ with $u=\left\langle a_{1,0}, b_{1,0}\right\rangle$ and $-u=\left\langle a_{1,0}, b_{1,0}\right\rangle$ correspond to the same representation of $K$, the last statement follows from Theorem 4.19 .

Example 5.2. We have seen in Remark 4.20 that the rep-polynomial of $C[2,3]$ and $C[1,2,2]$ are $u\left(u^{6}-u^{4}+2 u^{2}-1\right)$ and $u\left(u^{6}+3 u^{4}+2 u^{2}-1\right)$, respectively. We can check that

$$
u^{6}-u^{4}+2 u^{2}-1=\left(u^{3}+u^{2}-1\right)\left(u^{3}-u^{2}+1\right)
$$

and

$$
u^{6}+3 u^{4}+2 u^{2}-1=\left(u^{3}+u^{2}+2 u+1\right)\left(u^{3}-u^{2}+2 u-1\right) .
$$

Remark 5.3. There exist $2^{\frac{\alpha-1}{2}}$ number of polynomials $g(u) \in \mathbb{C}[u]$ such that $P_{K}(u)=$ $u g(u) \hat{g}(u)$ for a 2-bridge knot $K=S(\alpha, \beta)$. But Theorem 5.1 implies that we can find one among them which has integer coefficients.

Remark 5.4. Theorem 5.1 does not hold for the link case. For example, the rep-polynomial of $C[2,1,2]$, the Whitehead link, is

$$
P_{K}(u)=u^{4}\left(u^{4} \pm 2 u^{2}+2\right)=u^{4}\left(u^{2}+1 \pm i\right)\left(u^{2}-1 \pm i\right) .
$$

The sign in the above equation depends on the orientations of the two components of $K$. If we change the orientation of one of two components, then $u$ is changed to $\pm i u$. As this example shows, Riley polynomial $W_{12}\left(u^{2}\right)=\frac{1}{u^{2}} P_{K}(u)$ might have 0 as a root in link case.

Some rep-polynomials of links are decomposed into two polynomials with integer coefficients, but the two factors do not usually have the same property as $g(u)$ in Theorem 5.1 . The rep-polynomials of a link $K=C[2,1,4]$ are $P_{K}(u)$ or $P_{K}(i u)$, where

$$
P_{K}(u)=u^{2}\left(u^{6}-3 u^{4}+4 u^{2}-1\right)\left(u^{6}-u^{4}+1\right) .
$$


When $K$ is a knot, $\frac{1}{u} P_{K}(u) \in \mathbb{Z}[u]$ is a monic polynomial whose constant term is either 1 or -1 by Lemma 4.18 . So it is easy to show that $r^{2}$ is a unit in $\mathbb{Q}\left(r^{2}\right)$ for each nonzero root $r$ of $P_{K}(u)$, and we can show using Theorem 5.1 that $r \in \mathbb{Q}\left(r^{2}\right)$ as follows.

Proposition 5.5. For any 2-bridge knot $K$, each nonzero root $r$ of $P_{K}(u)$ belongs to the trace field $\mathbb{Q}\left(r^{2}\right)$, as a unit, of the parabolic representation corresponding to $r$.

Proof. Let $K=C\left[n_{1}, \cdots, n_{k}\right]$. By Lemma 4.18 , there is $c_{2 k} \in \mathbb{Z}, k=1,2, \cdots, \frac{\alpha-3}{2}$ such that

$$
P_{K}(u)=u\left(u^{\alpha-1}+c_{\alpha-3} u^{\alpha-3}+\cdots+c_{2} u^{2} \pm 1\right) .
$$

Therefore each nonzero root $r$ of $P_{K}(u)$ satisfies the following equation:

$$
r^{2}\left(r^{\alpha-3}+c_{\alpha-3} r^{\alpha-5}+\cdots+c_{2}\right)= \pm 1
$$

which implies that $r$ is a unit in $\mathbb{Q}\left(r^{2}\right)$ if $r \in \mathbb{Q}\left(r^{2}\right)$.

By Theorem 5.1, $P_{K}(u)= \pm u g(u) g(-u)$ and thus there are $A(u)$ and $B(u)$ in $\mathbb{Z}[u]$ such that $g(u)=A\left(u^{2}\right)+u B\left(u^{2}\right)$. Note that $\hat{g}(u) \neq g(u)$ and thus $B\left(u^{2}\right) \neq 0$. So if $g(r)=0$

then $r=-\frac{A\left(r^{2}\right)}{B\left(r^{2}\right)} \in \mathbb{Q}\left(r^{2}\right)$, which implies that $\mathbb{Q}(r)=\mathbb{Q}\left(r^{2}\right)$.

Remark 5.6. Proposition 5.5 does not hold for 2-bridge links. For example, the reppolynomial of $C[2,1,2]$ is $u^{4}\left(u^{4} \pm 2 u^{2}+2\right)$, and $\mathbb{Q}(r)$ is not equal to $\mathbb{Q}\left(r^{2}\right)$ for any $r$ such that $r^{4} \pm 2 r^{2}+2=0$.

Corollary 5.7. Let $C\left[n_{1}, n_{2}, \cdots, n_{k}\right]$ and $r$ be a non-zero root of the rep-polynomial $P_{K}(u)$ of $K$. Then $\mathbb{Q}(r)=\mathbb{Q}\left(u_{k}(r)\right)$.

\section{Complex volume And Cusp shape}

In this section, we will see that the complex volume and the cusp shape of a parabolic representation of an arbitrary 2-bridge knot can be easily computed using the quandle coloring.

Let $C\left[n_{1}, \cdots, n_{k}\right]$ be any Conway diagram of a 2-bridge knot $K$ with an arc coloring $\left\{a_{i, j}, b_{i, j}\right\}$ which corresponds to a parabolic representation $\rho: G(K) \rightarrow S L(2, \mathbb{C})$ and $n=n_{1}+\cdots+n_{k}$. Then we have $n+2$ regions, $r_{1}, \cdots, r_{n+2}$ and we can define a region coloring $\beta:\left\{r_{1}, \cdots, r_{n+2}\right\} \rightarrow \mathbb{C}^{2}$ on the given Conway diagram, satisfying the condition illustrated in Figure 12 around each arc [18. Now we choose any non-zero generic vector $p \in \mathbb{C}^{2}$ and assign a complex number $w_{j}=\left\langle p, \beta\left(r_{j}\right)\right\rangle$ to each region $r_{j}$, which is called $a$ region variable, and define a function $C\left(w_{1}, \cdots, w_{n+2}\right)$ by the sum of

$$
\begin{cases}\frac{w_{a} w_{c}-w_{b} w_{d}}{\left(w_{a}-w_{d}\right)\left(w_{c}-w_{b}\right)}-1 \text { for Figure } 13 \text { (left) } \\ \frac{w_{a} w_{c}-w_{b} w_{d}}{\left(w_{a}-w_{d}\right)\left(w_{c}-w_{b}\right)}+1 \text { for Figure } 13 \text { (right) }\end{cases}
$$

over all crossings [22]. The potential function $W\left(w_{1}, \cdots, w_{n+2}\right)$ which is defined by Cho 


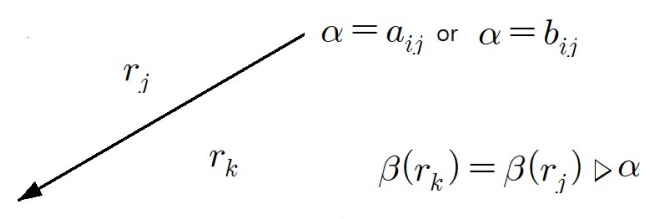

FiguRE 12. Rule for a region coloring
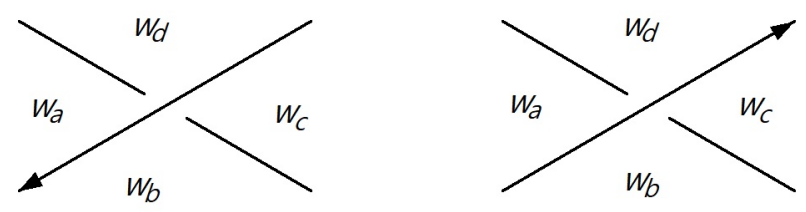

FiguRE 13. Region variables at a crossing

and Murakami in [6, 7], is the sum of

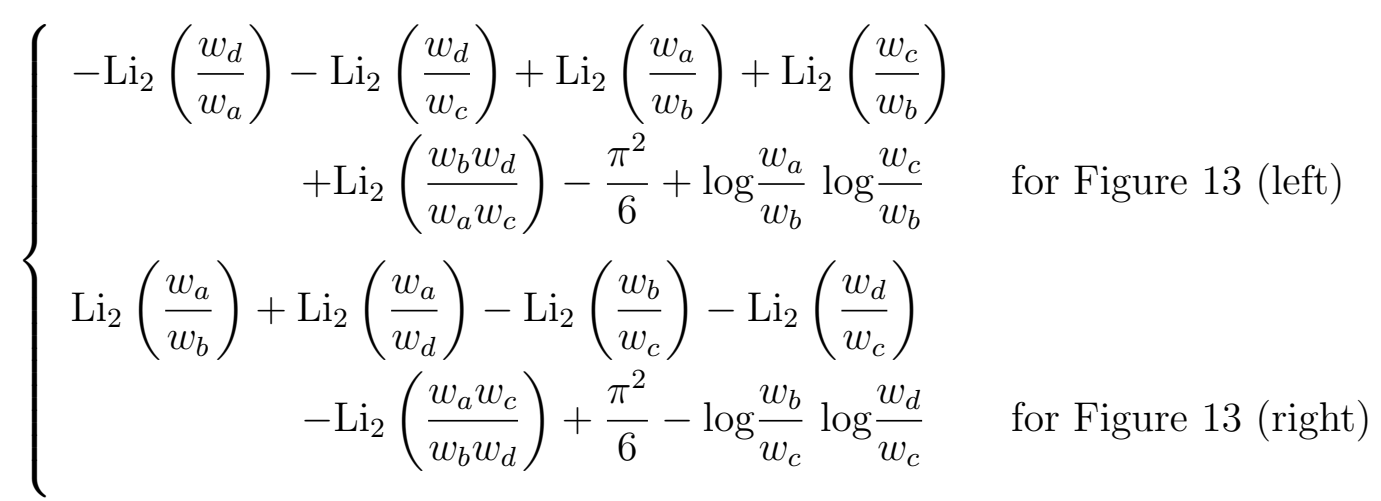

over all crossings.

Using the above two functions defined on the region variables induced from the arc coloring vectors, the cusp shape and the complex volume $\operatorname{Vol}_{\mathbb{C}}(\rho) \in \mathbb{C} / i \pi^{2} \mathbb{Z}$ can be easily computed as follows for any parabolic representation $\rho$ of $K$ [6, 7, 23]. (See also [40] and [13] for the earlier relevant works for complex volume.)

Theorem 6.1. Let $\left\{a_{i, j}, b_{i, j}\right\}$ be an arc coloring of a 2-bridge knot $K=C\left[n_{1}, \cdots, n_{k}\right]$ corresponding to a parabolic representation $\rho: G(K) \rightarrow S L(2, \mathbb{C})$. Let $\left\{w_{i}\right\}_{i=1, \cdots, n+2}$ be any region variable induced from the arc coloring, where $n=\sum_{j=1}^{k} n_{j}$. Then 
(i) the cusp shape of $\rho$ is given by $C\left(w_{1}, \cdots, w_{n+2}\right)$,

(ii) the complex volueme of $\rho$ is given by

$$
i \operatorname{Vol}_{\mathbb{C}}(\rho) \equiv W_{0}\left(w_{1}, \cdots, w_{n+2}\right) \quad\left(\bmod \pi^{2} \mathbb{Z}\right) .
$$

Here the function $W_{0}$ is defined as follows.

$$
W_{0}\left(w_{1}, \cdots, w_{n+2}\right):=W\left(w_{1}, \cdots, w_{n+2}\right)-\sum_{k=1}^{n+2}\left(w_{k} \frac{\partial W}{\partial w_{k}}\right) \log w_{k}
$$

Example 6.2. Let $K_{1}=C[2,3,0,3,2,-2,2,3]$ and $K_{2}=C[2,3]$. Then by Theorem 6.1, Proposition 6.2 and Remark 6.3 of [31, there is a proper branched fold map $f$ : $\left(\mathbb{S}^{3}, K_{1}\right) \rightarrow\left(\mathbb{S}^{3}, K_{2}\right)$ which respects the bridge structures, which induces an epimorphism $f_{*}: G\left(K_{1}\right) \rightarrow G\left(K_{2}\right)$, and the degree of $f_{*}$ is equal to 3 .

We have seen in Example 5.2 that the rep-polynomial $P_{\frac{3}{7}}(u)$ of $K_{2}$ is as follows:

$$
P_{\frac{3}{7}}(u)=u\left(u^{6}-u^{4}+2 u^{2}-1\right)=u\left(u^{3}+u^{2}-1\right)\left(u^{3}-u^{2}+1\right) .
$$

The rep-polynomial $P_{\frac{101}{217}}(u)$ of $K_{1}$ is expressed as

$$
P_{\frac{101}{217}}(u)=u\left(u^{3}+u^{2}-1\right)\left(u^{3}-u^{2}+1\right) h(u) \hat{h}(u),
$$

where $h(u)$ is an irreducible monic polynomial of degree 105:

$$
h(u)=u^{105}-2 u^{104}-5 u^{103}+14 u^{102}+\cdots-4 u^{2}+1 .
$$

We can observe that $P_{\frac{3}{7}}(u) \mid P_{\frac{101}{217}}(u)$. We will see in the next section that it is generally true that if $K_{1} \geq K_{2}$ then $P_{K_{2}}(u)$ divides either $P_{K_{1}}(u)$ or $P_{K_{1}}^{\prime}(u)$ (see Theorem 7.6).

Now we compute the cusp shapes and the complex volumes of the parabolic representations of $G\left(K_{1}\right)$ and $G\left(K_{2}\right)$ which correspond to the non-zero roots of $P_{\frac{3}{7}}(u)$ using Theorem 6.1. By the calculation using Mathematica, we can check that the cusp shapes and the complex volumes of $K_{1}$ are exactly 3 times those of $K_{2}$ as expected, respectively. (See Table 1 and Table 2, )

\begin{tabular}{|c|c|c|}
\hline non-zero roots of $P_{\frac{3}{7}}(u)$ & $K_{1}=C[2,3,0,3,2,-2,2,3]$ & $K_{2}=C[2,3]$ \\
\hline$u=0.75487766$ & -27.05853199 & -9.01951066 \\
\hline$u=0.87743883+0.74486176 i$ & $-7.47073400+8.93834119 i$ & $-2.49024466+2.97944706 i$ \\
\hline$u=0.87743883-0.74486176 i$ & $-7.47073400-8.93834119 i$ & $-2.49024466-2.97944706 i$ \\
\hline
\end{tabular}

TABLE 1 . cusp shapes of $K_{1}$ and $K_{2}$

Note that $\operatorname{cs}\left(K_{1}\right)=3 \operatorname{cs}\left(K_{2}\right)+4 \pi^{2}$ when $u=0.8774388331+0.7448617666 i$ and hence

$$
\operatorname{cs}\left(K_{1}\right)=3 \operatorname{cs}\left(K_{2}\right) \quad\left(\bmod \pi^{2} \mathbb{Z}\right) .
$$

(See Table 2.) 


\begin{tabular}{|c|c|c|}
\hline non-zero roots of $P_{\frac{3}{7}}(u)$ & $K_{1}=C[2,3,0,3,2,-2,2,3]$ & $K_{2}=C[2,3]$ \\
\hline$u=0.75487766$ & $0+3.34036365 i$ & $0+1.11345455 i$ \\
\hline$u=0.87743883+0.74486176 i$ & $-8.48436626+30.40603247 i$ & $-2.82812208-3.02412837 i$ \\
\hline$u=0.87743883-0.74486176 i$ & $8.48436626-9.07238513 i$ & $2.82812208-3.02412837 i$ \\
\hline
\end{tabular}

TABLE 2. complex volumes of $K_{1}$ and $K_{2}$

\section{EPIMORPHISMS BETWEEN KNOT GROUPS}

There is a partial order on the set of prime knots as follows : We write $K_{1} \geq K_{2}$ for two prime knots if there exists an epimorphism from $G\left(K_{1}\right)$ to $G\left(K_{2}\right)$. A knot is called minimal if its knot group admits epimorphisms onto the knot groups of only the trivial knot and itself.

7.1. ORS-expansion. Ohtsuki, Riley and Sakuma have constructed in [31] systemetically epimorphisms between 2-bridge knot groups preserving peripheral structure when the two knots have some special continued fraction expansions, and then it can be shown that all epimorphisms between 2-bridge knot groups arise only from those Ohtsuki-Riley-Sakuma construction by the result of Aimi-Lee-Sakai-Sakuma [2]. (See [36] and also [1] .) Therefore non-minimal 2-bridge knots have the following special Conway's normal forms.

Definition 7.1. We say $K$ has an $O R S$-expansion of type $n$ with respect to $\mathbf{a}=\left(a_{1}, a_{2}, \cdots, a_{m}\right)$ if $K$ can be written as

$$
K=C\left[\epsilon_{1} \mathbf{a}, 2 c_{1}, \epsilon_{2} \mathbf{a}^{-\mathbf{1}}, 2 c_{2}, \epsilon_{3} \mathbf{a}, 2 c_{3}, \epsilon_{4} \mathbf{a}^{-\mathbf{1}}, 2 c_{4}, \cdots, \epsilon_{n-1} \mathbf{a}^{-\mathbf{1}}, 2 c_{n-1}, \epsilon_{n} \mathbf{a}^{(-\mathbf{1})^{\mathbf{n}+\mathbf{1}}}\right]
$$

where

$$
\mathbf{a}^{-\mathbf{1}}=\left(a_{m}, a_{m-1}, \cdots, a_{1}\right), \epsilon_{i}= \pm 1\left(\epsilon_{1}=1\right), c_{i} \in \mathbb{Z} .
$$

Remark 7.2. Let $K$ be an ORS-expansion of type $n$ with respect to $\mathbf{a}=\left(a_{1}, a_{2}, \cdots, a_{m}\right)$. Then

(1) $K$ is a knot if $n$ is odd and it is a link if $n$ is even.

(2) We can exclude the case $c_{i}=0, \epsilon_{i} \epsilon_{i+1}=-1$, and this expansion with respect to a is unique (see [36] and [12] for details).

(3) If $c_{i}=0$ and $\epsilon_{i} \epsilon_{i+1}=1$, then we can reduce the length of the expansion by 2 . Thus the resulting expansion after doing all the possible reducing, is the reduced even expansion of $K$, if a is a reduced even expansion.

(4) $C\left[a_{m}, a_{m-1}, \cdots, a_{1}\right]$ is equivalent to $C\left[a_{1}, a_{2}, \cdots, a_{m}\right]$ if $m$ is odd and it is equivalent to the mirror of $C\left[a_{1}, a_{2}, \cdots, a_{m}\right]$ if $m$ is even. It follows from the fact the upsidedown of $C\left[a_{1}, a_{2}, \cdots, a_{m}\right]$ is $C\left[(-1)^{m+1} a_{m},(-1)^{m+1} a_{m-1}, \cdots,(-1)^{m+1} a_{1}\right]$.

Theorem 7.3. Let $K$ be a 2-bridge link which has an ORS-expansion of type $n$ with respect to $\mathbf{a}=\left(a_{1}, a_{2}, \cdots, a_{m}\right)$. Suppose that $\left(u_{1}, \cdots, u_{m}\right)$ is the $u_{i}$-sequence of $A=$ $C\left[a_{1}, a_{2}, \cdots, a_{m}\right]$. Then 

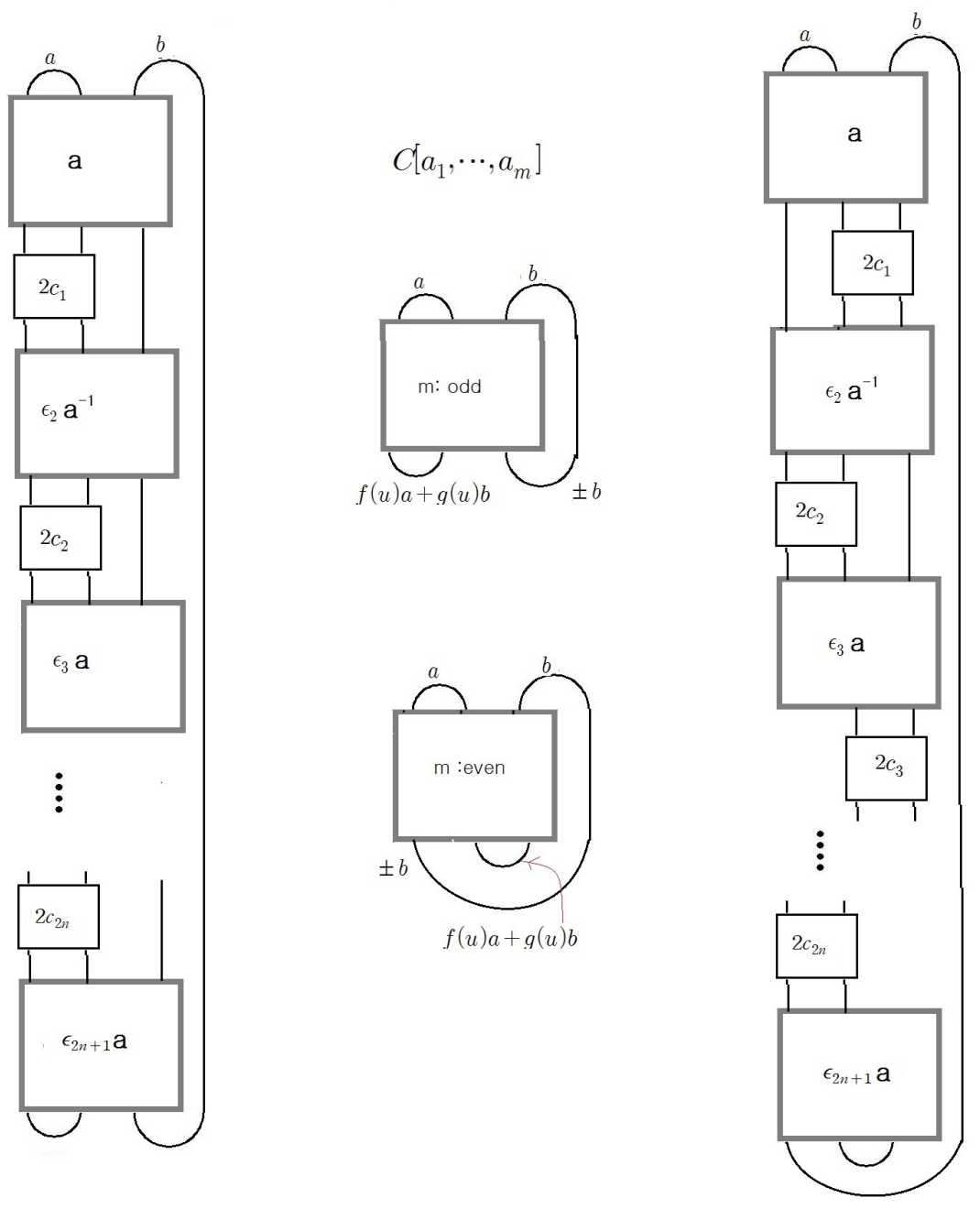

Figure 14. $C\left[\epsilon_{1} \mathbf{a}, 2 c_{1}, \epsilon_{2} \mathbf{a}^{-\mathbf{1}}, 2 c_{2}, \epsilon_{3} \mathbf{a}, 2 c_{3}, \cdots, 2 c_{2 n}, \epsilon_{2 n+1} \mathbf{a}\right]:$ knots

(i) the rep-polynomial $P_{K}(u)$ of $K$ has the rep-polynomial $P_{A}(u)$ of $A$ as a factor if $K$ is a knot, and either $P_{K}(u)$ or $P_{K}(i u)$ has $P_{A}(u)$ as a factor if $K$ is a link.

(ii) the $u_{i}$-sequence of $K$ is as follows when it is considered in $\mathbb{Z}[u] /\left(\frac{1}{u} P_{A}(u)\right)$.

$$
\left(u_{1}, \cdots, u_{m}, 0, \pm u_{m}, \cdots, \pm u_{1}, 0, \pm u_{1}, \cdots, \pm u_{m}, 0, \cdots, 0, \pm u_{1}, \cdots, \pm u_{m}\right)
$$

where the sign is not determined.

Proof. We may assume that the orientation of $C\left[a_{1}, a_{2}, \cdots, a_{m}\right]$ is the same as the first part's orientation of $K$, by changing the orientation of one component of a link $K$ if necessary, because each link has two rep-polynomials up to its orientation and one is obtained from the other by converting $u$ to $i u$. 
Consider a $\mathfrak{C}$-coloring on $K$ which starts with two vectors $a, b$ such that $u=\langle a, b\rangle$ is a root of $P_{A}(u)$. Then the last two vectors of $C\left[a_{1}, a_{2}, \cdots, a_{m}\right]$ are $f(u) a+g(u) b, \pm b$ for some polynomials $f(u)$ and $g(u)$, and all the vectors of $2 c_{1}$-block are $\pm(f(u) a+g(u) b)$ by Lemma 4.1. (See Figure 14.) So the first two vectors of $\epsilon_{2} \mathbf{a}^{-\mathbf{1}}$-blocks are $\pm(f(u) a+g(u) b), \pm b$. We claim that the last two vectors of $\epsilon_{2} \mathbf{a}^{-\mathbf{1}}$-blocks are $\pm\left(a+h_{2}(u) b\right), \pm b$ for some $h_{2}(u) \in \mathbb{Z}[u]$. If $\epsilon_{2}=-1$, then these blocks are the horizontally reflected diagram of $\epsilon_{1}$ a-block with an reversed orientation and thus all the coloring vectors are also reflected up to sign and especially the last two vectors are $\pm a, \pm b$. If $\epsilon_{2}=1$, then these blocks are the horizontally half-rotated diagram of $\epsilon_{1}$ a-blocks with the reversed orientation and thus by Lemma 4.16 and Proposition 4.17 there is such $h_{2}(u) \in \mathbb{Z}[u]$. See Figure 15 .
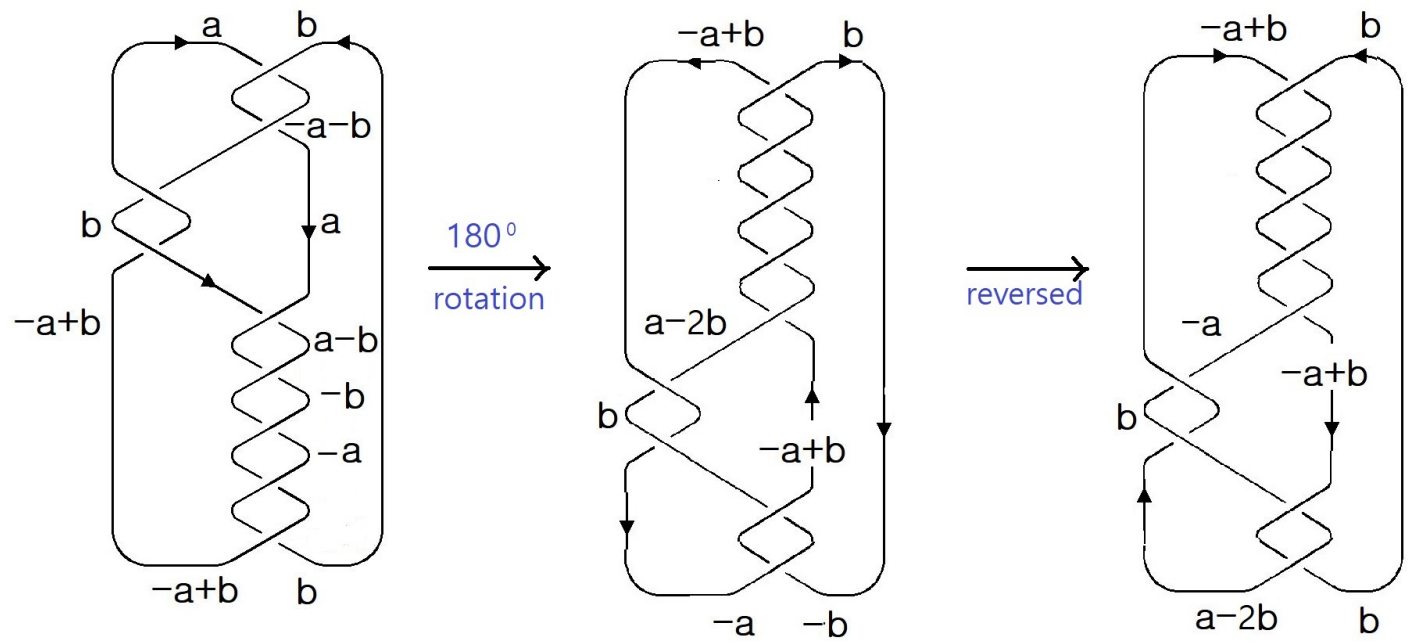

FiguRE 15. when $\epsilon_{2}=1 ;\langle a, b\rangle=1$

By Lemma 4.1 again, all the vectors of $2 c_{2}$-block are $\pm\left(a+h_{2}(u)\right) b$ and thus the first two vectors of $\epsilon_{3}$ a-block are $\pm\left(a+h_{2}(u)\right) b, \pm b$. Since $\left\langle a+h_{2}(u) b, b\right\rangle=\langle a, b\rangle=u$, the last two vectors of $\epsilon_{3}$ a-block must be of the form $f_{3}(u) a+g_{3}(u) b, \pm b$ for some $f_{3}(u), g_{3}(u) \in \mathbb{Z}[u]$ satisfying

$$
u_{m}(u)= \pm\langle f(u) a+g(u) b, b\rangle= \pm\left\langle f_{3}(u) a+g_{3}(u) b, b\right\rangle
$$

(We see that $f_{3}(u)$ is necessarily equal to either $f(u)$ or $-f(u)$.)

By repeating this process, we can conclude that the last two vectors of the diagram

$$
C\left[\epsilon_{1} \mathbf{a}, 2 c_{1}, \epsilon_{2} \mathbf{a}^{-\mathbf{1}}, 2 c_{2}, \epsilon_{3} \mathbf{a}, 2 c_{3}, \epsilon_{4} \mathbf{a}^{-\mathbf{1}}, 2 c_{4}, \cdots, \epsilon_{2 n-1} \mathbf{a}, 2 c_{2 n-1}, \epsilon_{2 n} \mathbf{a}^{-\mathbf{1}}\right]
$$

are $\pm\left(a+h_{2 n}(u) b\right), \pm b$, and the last two vectors of the diagram

$$
C\left[\epsilon_{1} \mathbf{a}, 2 c_{1}, \epsilon_{2} \mathbf{a}^{-\mathbf{1}}, 2 c_{2}, \epsilon_{3} \mathbf{a}, 2 c_{3}, \epsilon_{4} \mathbf{a}^{-\mathbf{1}}, 2 c_{4}, \cdots, \epsilon_{2 n} \mathbf{a}^{-\mathbf{1}}, 2 c_{2 n}, \epsilon_{2 n+1} \mathbf{a}\right]
$$

are $f_{2 n+1}(u) a+g_{2 n+1}(u) b, \pm b$ such that

$$
\langle f(u) a+g(u) b, b\rangle= \pm\left\langle f_{2 n+1}(u) a+g_{2 n+1}(u) b, b\right\rangle .
$$


This implies that $u$ is also a root of the rep-polynomial of $K$, which implies that $P_{A}(u)$ divides $P_{K}(u)$. This proves (i).

The statement (ii) is obvious from Lemma 4.1, Lemma 4.16, and Lemma 4.17.

Example 7.4. A link $C[3,2,3]$ and a knot $C[3,2,6]=C[3,2,3,0,3]$ are ORS-expansions of type 2 and 3 with respect to $\mathbf{a}=(3)$. Therefore the rep-polynomial of $C[3,2,6]$ is divided by the rep-polynomial of $C[3], u\left(u^{2}-1\right)$, and so is the rep-polynomial of $C[3,2,3]$ if we choose a suitable orientation. These are the first 2 diagrams of Figure 16. We can check that if
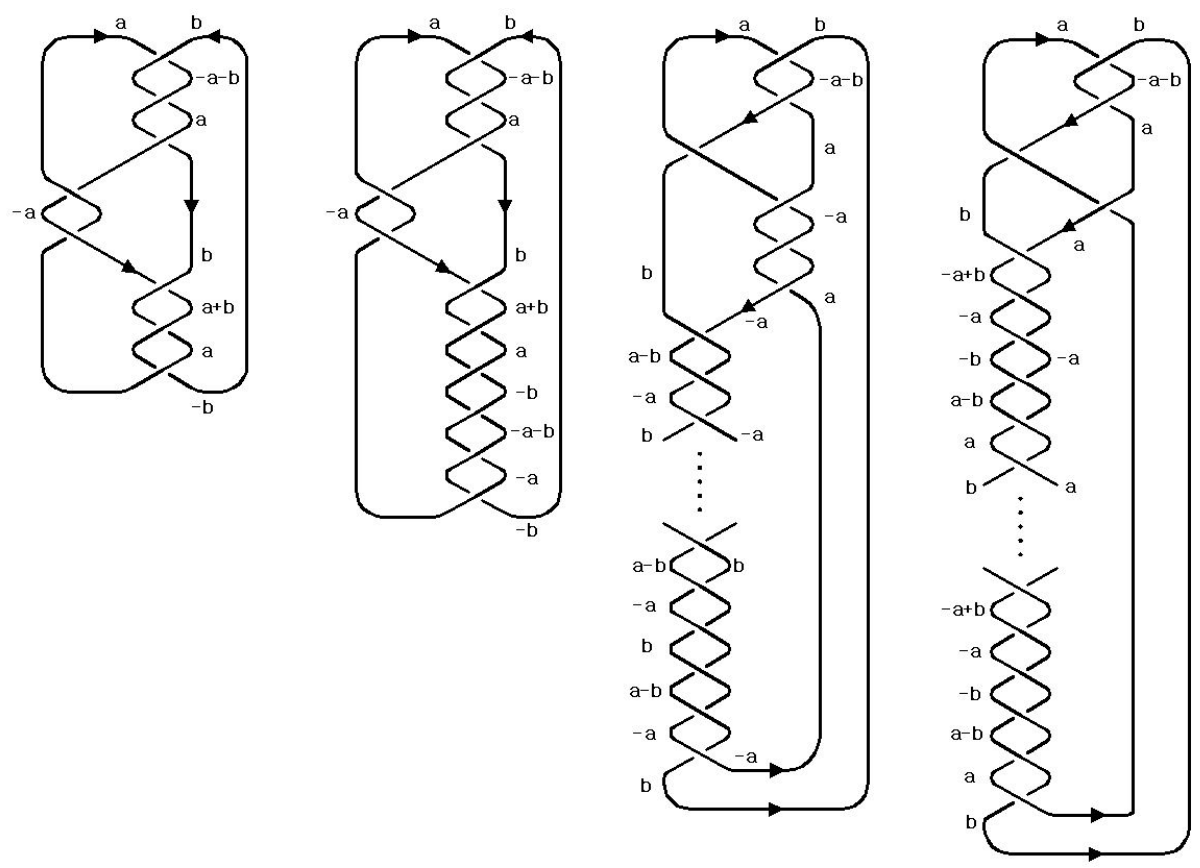

Figure 16. Knots or links $\geq 3_{1} ;\langle a, b\rangle=1$

the orientation of one component of a link $C[3,2,3]$ is reversed, then $i P_{C[3]}(i u)=u\left(u^{2}+1\right)$ divides the rep-polynomial $P_{K}(u)$ of $K=C[3,2,3]$, or equivalently, $P_{C[3]}(u)$ divides $P_{K}(i u)$. (See Figure 17.) Note that the two rep-polynomials of $K=C[3,2,3]$ is

$$
P_{1}(u)=u^{2}\left(u^{2}-1\right)^{3}\left(u^{2}-2\right)\left(u^{6}-3 u^{4}+2 u^{2}+2\right)\left(u^{8}-2 u^{6}+2 u^{2}+1\right)
$$

and

$$
P_{2}(u)=u^{2}\left(u^{2}+1\right)^{3}\left(u^{2}+2\right)\left(u^{6}+3 u^{4}+2 u^{2}-2\right)\left(u^{8}+2 u^{6}-2 u^{2}+1\right),
$$

and $u\left(u^{2}-1\right)$ is a factor of $P_{1}(u)= \pm P_{2}(i u)$.

Kitano and Morifuji proved in 24] that $K_{1} \geq K_{2}$ if the Riley polynomial of $K_{2}$ divides that of $K_{1}$. Therefore the following Ohtsuki-Riley-Sakuma's result immediately follows from Theorem 7.3 . 


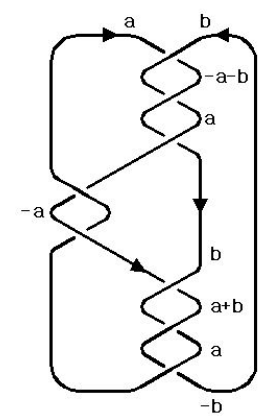

$\langle a, b\rangle=1$

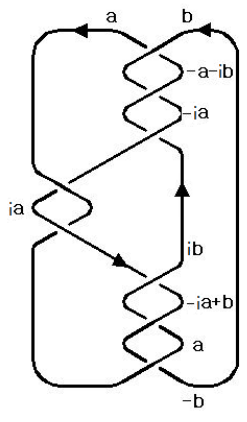

$\langle a, b\rangle=i$

Figure 17. $C[3,2,3]-\operatorname{link} \geq 3_{1}$

Corollary 7.5 (Ohtsuki-Riley-Sakuma, [31]). Let $K$ be a 2-bridge knot or link which has an ORS-expansion of type $n$ with respect to $\mathbf{a}=\left(a_{1}, a_{2}, \cdots, a_{m}\right)$. Then $K \geq A=$ $C\left[a_{1}, a_{2}, \cdots, a_{m}\right]$.

7.2. Epimorphisms and Riley polynomials. In this subsection, we prove the converse statement of the result of Kitano and Morifuji. To do this, we need to prove the following.

Theorem 7.6. Let $K_{1}$ and $K_{2}$ be 2-bridge knots. Then the followings are equivalent.

(i) $K_{1} \geq K_{2}$.

(ii) $P_{K_{2}}(u)$ divides either $P_{K_{1}}(u)$ or $P_{K_{1}}^{\prime}(u)$.

In the case that $K_{1} \geq K_{2}$ and $K_{1}$ is a link ( $K_{2}$ could be a knot or link), $P_{K_{2}}(u)$ divides one of the four rep-polynomials of $K_{1}, P_{K_{1}}(u), P_{K_{1}}(i u), P_{K_{1}}^{\prime}(u), P_{K_{1}}^{\prime}(i u)$.

Proof. Let $K_{2}=C\left[a_{1}, \cdots, a_{m}\right]$ and $\mathbf{a}=\left(a_{1}, \cdots, a_{m}\right)$.

(ii) $\Rightarrow$ (i) : Since if the rep-polynomial of $K_{2}=C\left[a_{1}, \cdots, a_{m}\right]$ is a factor of a reppolynomial of $K_{1}=C\left[n_{1}, \cdots, n_{k}\right]$ then the Riley polynomial of $K_{2}=S\left(\alpha_{2}, \beta_{2}\right)$ is a factor of the Riley polynomial of $K_{1}=S\left(\alpha_{1}, \beta_{1}\right)$ with $\frac{\beta_{1}}{\alpha_{1}}=\left[n_{1}, \cdots, n_{k}\right]$ and $\frac{\beta_{2}}{\alpha_{2}}=\left[a_{1}, \cdots, a_{m}\right]$, there exists an epimorphism from $G\left(K_{1}\right)$ to $G\left(K_{2}\right)$ by the result of Kitano and Morifuji, Theorem 3.1 in [24].

(i) $\Rightarrow$ (ii) : If we assume that there exists an epimorphism from $G\left(K_{1}\right)$ to $G\left(K_{2}\right)$, then $K_{1}$ has an ORS-expansion of type $2 n+1$ with respect to any Conway's normal form $K_{2}=C[\mathbf{a}]$, that is,

$$
K_{1}=C\left[\epsilon_{1} \mathbf{a}, 2 c_{1}, \epsilon_{2} \mathbf{a}^{-\mathbf{1}}, 2 c_{2}, \epsilon_{3} \mathbf{a}, 2 c_{3}, \epsilon_{4} \mathbf{a}^{-\mathbf{1}}, 2 c_{4}, \cdots, \epsilon_{2 n} \mathbf{a}^{-\mathbf{1}}, 2 c_{2 n}, \epsilon_{2 n+1} \mathbf{a}\right]
$$

where

$$
\mathbf{a}^{-\mathbf{1}}=\left(a_{m}, a_{m-1}, \cdots, a_{1}\right), \epsilon_{i}= \pm 1\left(\epsilon_{1}=1\right), c_{i} \in \mathbb{Z}
$$

by [2] and [31] (see also Theorem 3.1 of [36]). Let

$$
\mathbf{b}=\left(\epsilon_{1} \mathbf{a}, 2 c_{1}, \epsilon_{2} \mathbf{a}^{-\mathbf{1}}, 2 c_{2}, \epsilon_{3} \mathbf{a}, 2 c_{3}, \epsilon_{4} \mathbf{a}^{-\mathbf{1}}, 2 c_{4}, \cdots, \epsilon_{2 n} \mathbf{a}^{-\mathbf{1}}, 2 c_{2 n}, \epsilon_{2 n+1} \mathbf{a}\right) .
$$


Then $P_{C[\mathbf{a}]}(u)$ divides $P_{C[\mathbf{b}]}(u)$ by Theorem 7.3, which implies that (ii) holds because $P_{C[\mathbf{b}]}(u)$ is either $P_{K_{1}}(u)$ or $P_{C[\mathbf{b}]}^{\prime}(u)$.

The last statement is similarly proved.

Corollary 7.7. Let $K_{1}=S(\alpha, \beta)$ and $K_{2}=S\left(\alpha^{\prime}, \beta^{\prime}\right)$. Then $K_{1} \geq K_{2}$ if and only if the Riley polynomial of $S\left(\alpha^{\prime}, \beta^{\prime}\right)$ divides the Riley polynomial of either $S(\alpha, \beta)$ or $S\left(\alpha, \beta^{\prime \prime}\right)$, where $\beta \beta^{\prime \prime} \equiv \pm 1(\bmod \alpha)$.

Proof. This follows from that if $\frac{\beta}{\alpha}=\left[n_{1}, n_{2}, \cdots, n_{k}\right]$ and $\frac{\beta^{\prime \prime}}{\alpha^{\prime \prime}}=\left[n_{k}, n_{k-1}, \cdots, n_{1}\right]$, then $\alpha=\alpha^{\prime \prime}$ and $\beta \beta^{\prime \prime} \equiv(-1)^{k-1}(\bmod \alpha)$.

Example 7.8. We have seen in Example 4.8 and Example 4.24 that the rep-polynomial of $K_{1}=C[2,2,5]$ and the trefoil $K_{2}=C[3]$ are

$$
P_{K_{1}}(u)=u(u-1)(u+1) h(u) h(-u)
$$

and

$$
P_{K_{2}}=u(u-1)(u+1)
$$

respectively, where

$$
h(u)=u^{12}-2 u^{10}+u^{9}+4 u^{8}-u^{7}-3 u^{6}+3 u^{5}+3 u^{4}-u^{3}-u^{2}+2 u+1 .
$$

Therefore $K_{1} \geq 3_{1}$ and $K_{1}$ has an ORS-expansion of type 3 with respect to $\mathbf{a}=(3)$, and also has an ORS-expansion of type 3 with respect to $\mathbf{a}=(2,-2)$. Actually,

$$
\begin{aligned}
K_{1} & =C[2,2,5]=C[3,-2,6]=C[3,-2,3,0,3] \\
& =C[2,-4,2,-2,2,-2]=C[2,-2,0,-2,2,-2,2,-2] .
\end{aligned}
$$

The upside-down diagram of $C[2,2,5]$ is

$$
C[5,2,2]=C[6,-2,3]=C[3,0,3,-2,3]=C[2,-2,2,-2,2,0,2,-2],
$$

and its rep-polynomial is $u(u-1)(u+1) \tilde{h}(u) \tilde{h}(-u)$, where

$$
\tilde{h}(u)=u^{12}+2 u^{11}-6 u^{10}-13 u^{9}+10 u^{8}+27 u^{7}-u^{6}-19 u^{5}-7 u^{4}+3 u^{3}+5 u^{2}+4 u+1 .
$$

Note that $h(u) \neq \tilde{h}(u)$, but they give the same trace field by Corollary 5.7 .

Remark 7.9. It is an immediate consequence of Theorem 7.6 that a 2-bridge knot $K$ is minimal if $K$ has an irreducible Riley polynomial. But the converse statement of this is not always true. For example, the double twist knot $J(4,4)$ is minimal (see Proposition 3.1 of [28]), but its rep-polynomial is

$$
P_{K}(u)=u\left(u^{3}+2 u+1\right)\left(u^{3}+2 u-1\right)\left(u^{4}+u^{3}+2 u^{2}+2 u+1\right)\left(u^{4}-u^{3}+2 u^{2}-2 u+1\right),
$$

which implies that the Riley polynomial of $J(4,4)$ has two irreducible factors. 


\section{APPEndix A. TORUS KNOTS AND LINKS}

A.1. Torus knots. To compute the rep-polynomial of the torus knot $K=T(2,2 k+1)$, we start with two vectors $a_{0}, b_{0}$ with $\left\langle a_{0}, b_{0}\right\rangle=u$. Then the vectors at the $i$-th step is calculated as follows. (See Figure 18.)

$$
\left(a_{i}, b_{i}\right)=\left(a_{0}, b_{0}\right) X(u)^{i}=\left(a_{0}, b_{0}\right)\left(\begin{array}{cc}
-p_{i-1}(-u) & -p_{i}(-u) \\
p_{i}(-u) & p_{i+1}(-u)
\end{array}\right)
$$

From this we get the last vectors

$$
a_{2 k+1}=-p_{2 k}(-u) a_{1}+p_{2 k+1}(-u) b_{1}
$$

and

Therefore

$$
b_{2 k+1}=-p_{2 k+1}(-u) a_{1}+p_{2 k+2}(-u) b_{1} .
$$

$$
P_{K}(u)= \pm\left\langle a_{0}, a_{2 k+1}\right\rangle= \pm u p_{2 k+1}(-u)= \pm\left\langle b_{0}, b_{2 k+1}\right\rangle
$$

and thus the solutions of $p_{2 k+1}(u)=0$ give non-abelian parabolic representations of $K=$ $T(2,2 k+1)$. By Lemma 2.1, this equation can be rewritten using $f_{n}(u):=p_{n+1}(u)-p_{n}(u)$ as follows.

$$
\begin{aligned}
p_{2 k+1}(u) & =p_{k+1}^{2}(u)-p_{k}^{2}(u) \\
& =\left(p_{k+1}(u)+p_{k}(u)\right)\left(p_{k+1}(u)-p_{k}(u)\right) \\
& =(-1)^{k} f_{k}(u) f_{k}(-u)=f_{k}(u) \hat{f}_{k}(u) \\
& =(-1)^{k} f_{k}\left(2-u^{2}\right)=\hat{f}_{k}\left(u^{2}-2\right) .
\end{aligned}
$$

So there is a 1-1 correspondence between the roots of $f_{k}(u)$ and the non-abelian parabolic representations of $K=T(2,2 k+1)$, and the Riley polynomial is $\pm f_{k}(2-y)$.

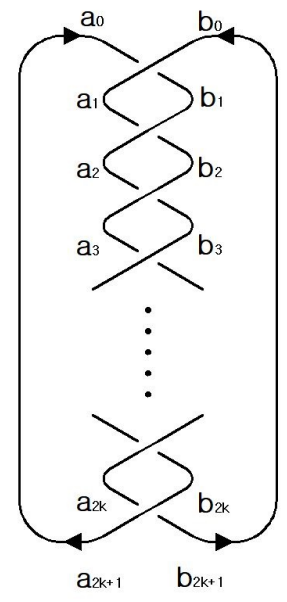

Torus knots
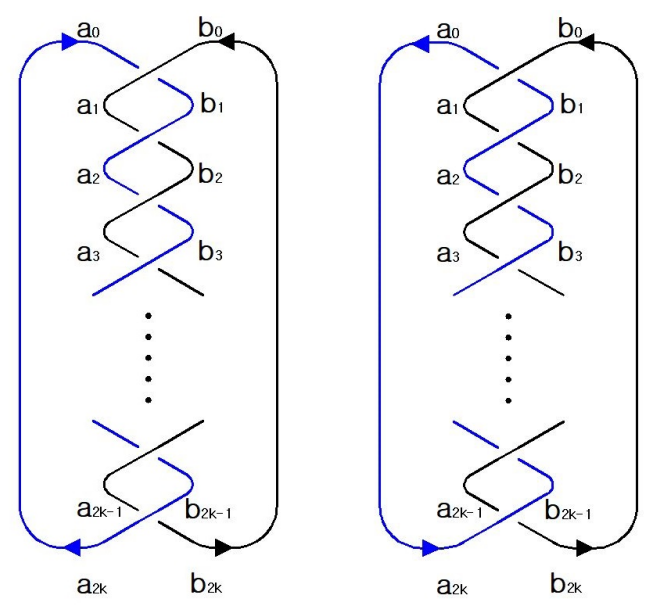

Torus links

FiguRe 18. Torus knots and Torus links 
A.2. Torus links : $K=T(2,2 k)$.

- Case 1: The left diagram of Figure 18.

From

$$
\left(a_{2 k}, b_{2 k}\right)=\left(a_{0}, b_{0}\right) X(u)^{2 k}=\left(a_{0}, b_{0}\right)\left(\begin{array}{cc}
-p_{2 k-1}(-u) & -p_{2 k}(-u) \\
p_{2 k}(-u) & p_{2 k+1}(-u)
\end{array}\right),
$$

we get the last two vectors

$$
a_{2 k}=-p_{2 k-1}(-u) a_{0}+p_{2 k}(-u) b_{0}
$$

and

$$
b_{2 k}=-p_{2 k}(-u) a_{0}+p_{2 k+1}(-u) b_{0} .
$$

Therefore

$$
P_{K}(u)= \pm\left\langle a_{0}, a_{2 k}\right\rangle= \pm u p_{2 k}(-u)= \pm\left\langle b_{0}, b_{2 k}\right\rangle
$$

Note that $u$ is a factor of $p_{2 k}(-u)$ and each root of

$$
\frac{1}{u^{2}} P_{K}(u)=\frac{1}{u} p_{2 k}(u)=0
$$

gives a non-abelian parabolic representation of $K=T(2,2 k)$. Since $p_{2}(u)=u$, there is no non-abelian parabolic representation for a link $K=T(2,2)$.

- Case 2 : The right diagram of Figure 18 .

If we let $t=-\left(2+u^{2}\right)$, then

$$
\begin{aligned}
\left(a_{2 k}, b_{2 k}\right) & =\left(a_{0}, b_{0}\right)(X(u) X(-u))^{k} \\
& =\left(a_{0}, b_{0}\right)\left(\begin{array}{cc}
-\left(p_{k-1}(t)+p_{k}(t)\right) & -u p_{k}(t) \\
-u p_{k}(t) & \left(p_{k}(t)+p_{k+1}(t)\right)
\end{array}\right),
\end{aligned}
$$

and thus we get the last vectors

$$
a_{2 k}=-\left(p_{k-1}(t)+p_{k}(t)\right) a_{0}-u p_{k}(t) b_{0}
$$

and

$$
b_{2 k}=-u p_{k}(t) a_{0}+\left(p_{k}(t)+p_{k+1}(t)\right) b_{0} .
$$

Therefore

$$
P_{K}(u)= \pm\left\langle a_{0}, a_{2 k}\right\rangle= \pm u^{2} p_{k}(t)= \pm u^{2} p_{k}\left(-2-u^{2}\right)= \pm\left\langle b_{0}, b_{2 k}\right\rangle
$$

Note that $p_{k}(-2)=(-1)^{k+1} k \neq 0$ by (ii) of Lemma 2.1 and this implies that $u$ is not a factor of $p_{k}\left(-2-u^{2}\right)$ and thus all the solutions of $\frac{P_{K}(u)}{u^{2}}=0$ give non-abelian parabolic representations of $K=T(2,2 k)$. Since $p_{1}\left(-2-u^{2}\right)=1$, we can check again that there is no non-abelian parabolic representation for a link $K=T(2,2)$.

Remark A.1. (i) From the above result we see that every torus link has non-abelian parabolic representations if it is not the Hopf link. For example, $K=T(2,4)$ has 2 non-abelian parabolic representations up to conjugate, which correspond to the roots of the equation $u^{2}= \pm 2$. Here $u^{2}-2=\frac{1}{u} p_{4}(u)$ is from Case 1 and $u^{2}+2=-p_{2}\left(-2-u^{2}\right)$ is from Case 2 . 
(ii) Since $p_{m n}(u)=p_{m}\left(v_{n}(u)\right) p_{n}(u), \forall m, n \in \mathbb{Z}$ by (vii) of Lemma 2.1, we get

$$
p_{2 k}(u)=p_{k}\left(v_{2}(u)\right) p_{2}(u)=u p_{k}\left(v_{2}(u)\right)
$$

and this implies the following: If we denote the two rep-polynomial of $K$ by $P_{1}(u)=$ $u p_{2 k}(u)$ and $P_{2}(u)=u^{2} p_{k}\left(-2-u^{2}\right)$, then

$$
P_{2}(i u)=-u^{2} p_{k}\left(-2+u^{2}\right)=-u^{2} p_{k}\left(v_{2}(u)\right)=-u p_{2 k}(u)=-P_{1}(u)
$$

and

$$
P_{1}(i u)=i u p_{2 k}(i u)=i u(i u) p_{k}\left(v_{2}(i u)\right)=-u^{2} p_{k}\left(-u^{2}-2\right)=-P_{2}(u) .
$$

Remark A.2. For each oriented Conway diagram $C\left[n_{1}, \cdots, n_{k}\right]$ of a 2-bridge link $K$, we can compute easily the rep-polynomial of $K$ applying the same procedure to each block as done in the torus link. That is, we get the last two vectors of the $i$-th block, $\epsilon_{i} a_{i, f}, \epsilon_{i}^{\prime} b_{i, f}$, multiplying the matrix $\left(\begin{array}{cc}-p_{n_{i}-1}(-u) & -p_{n_{i}}(-u) \\ p_{n_{i}}(-u) & p_{n_{i}+1}(-u)\end{array}\right)$ to the first two vectors of the $i$-th block, $\epsilon_{i} a_{i, 0}, \epsilon_{i}^{\prime} b_{i, 0}$. Here $\epsilon_{i}$ and $\epsilon_{i}^{\prime}$ are either 1 or $i$ depending upon the orientation of the arcs.

Appendix B. $J\left(2 n_{1}, 2 n_{2}, \cdots, 2 n_{m}\right)$-KNOTS AND LINKS

Each 2-bridge link $K$ has an even expansion $J\left(2 n_{1}, 2 n_{2}, \cdots, 2 n_{m}\right)$, where $m$ is even if $K$ is a knot and $m$ is odd if $K$ is a link. In this section, we compute the rep-polynomial of such diagrams. Using the even expansions, we don't need to consider $\epsilon_{i}, \epsilon_{i}^{\prime}$ for each block and thus we have an explicit formula for the matrix to be multiplied at each block only depending upon whether $i$ is even or odd, that is, whether the block is on the left line or on the right line.
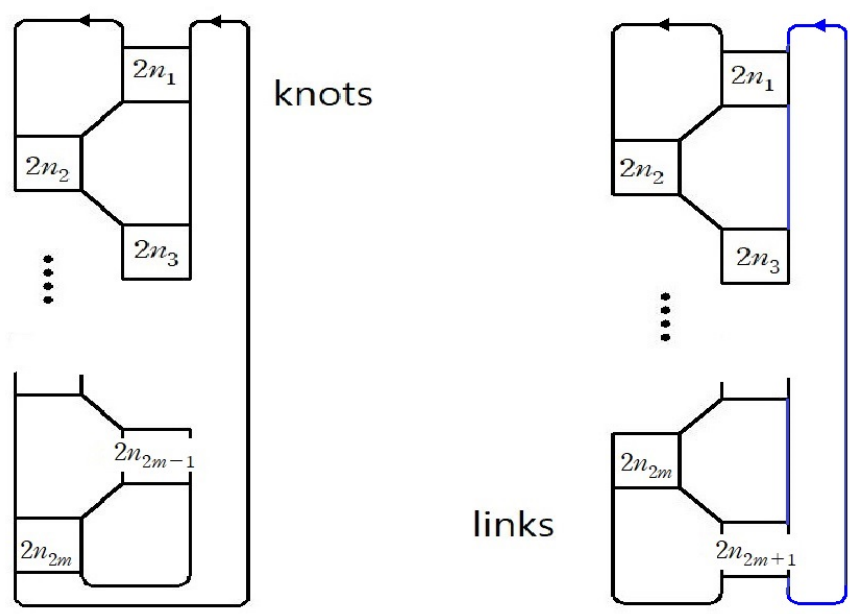

FiguRE 19. even expansion diagram

From our convention of indexing in the diagram,

$$
a_{2,0}=a_{1,0}, \quad b_{2,0}=a_{1, f}
$$


and for $k \geq 1$

$$
a_{2 k+1,0}=b_{2 k, f}, b_{2 k+1,0}=b_{2 k-1, f}, a_{2 k+2,0}=a_{2 k, f}, b_{2 k+2,0}=a_{2 k+1, f} .
$$

If we give an orientation on this diagram, then all the blocks on the right lines have the same orientation types and the same is true for all the blocks on the left lines. For example, if the orientation of $b_{1,0}$ is given as in Figure 19, then the orientation of each block will be as in Figure 20.
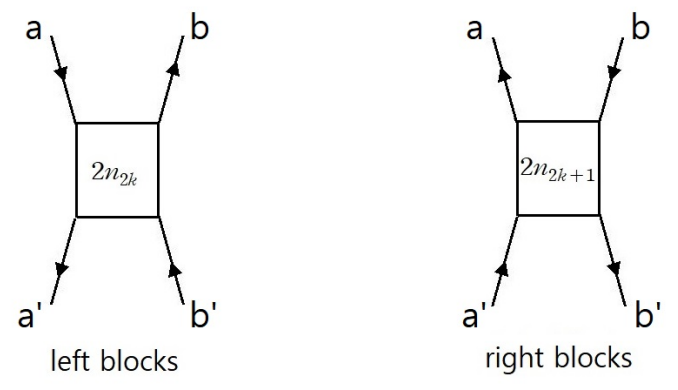

Figure 20.

Therefore we can compute arc vectors in each block as follows.

(1) If $a=a_{2 k, 0}, b=b_{2 k, 0}$ and $a^{\prime}=a_{2 k, f}, b^{\prime}=b_{2 k, f}$, then we get

$$
\begin{aligned}
\left(a^{\prime}, b^{\prime}\right)=(a, b)(X(-u) X(u))^{n} & =(a, b)\left(\begin{array}{cc}
-\left(p_{n-1}(t)+p_{n}(t)\right) & u p_{n}(t) \\
u p_{n}(t) & \left(p_{n}(t)+p_{n+1}(t)\right)
\end{array}\right) \\
& =(a, b)\left(\begin{array}{cc}
\left.(-1)^{n} f_{n-1}(-t)\right) & u p_{n}(t) \\
u p_{n}(t) & \left.(-1)^{n} f_{n}(-t)\right)
\end{array}\right)
\end{aligned}
$$

where $u=u_{2 k}, t=t_{2 k}=-2-u_{2 k}^{2}, n=n_{2 k}$.

(2) If $a=a_{2 k+1,0}, b=b_{2 k+1,0}$ and $a^{\prime}=a_{2 k+1, f}, b^{\prime}=b_{2 k+1, f}$ and $k>0$, then we get

$$
\begin{aligned}
\left(a^{\prime}, b^{\prime}\right)=(a, b)(X(u) X(-u))^{n} & =(a, b)\left(\begin{array}{cc}
-\left(p_{n-1}(t)+p_{n}(t)\right) & -u p_{n}(t) \\
-u p_{n}(t) & \left(p_{n}(t)+p_{n+1}(t)\right)
\end{array}\right) \\
& =(a, b)\left(\begin{array}{cc}
\left.(-1)^{n} f_{n-1}(-t)\right) & -u p_{n}(t) \\
-u p_{n}(t) & \left.(-1)^{n} f_{n}(-t)\right)
\end{array}\right)
\end{aligned}
$$

where $u=u_{2 k+1}, t=t_{2 k+1}=-2-u_{2 k+1}^{2}, n=n_{2 k+1}$.

By the above process, we obtain the last two vectors $a_{m, f}, b_{m, f}$ and then we get the reppolynomial $P_{K}(u)$ of $K$ as follows. If $K$ is a knot, which is the case when $m$ is even, $P_{K}(u)= \pm\left\langle a_{m, f}, b_{1,0}\right\rangle$, and $P_{K}(u)= \pm\left\langle b_{m, f}, b_{1,0}\right\rangle$ if $K$ is a link.

\section{REFERENCES}

[1] I. Agol, The classification of non-free 2-parabolic generator Kleinian groups, Slides of talks given at Austin AMS Meeting and Budapest Bolyai conference, July 2002, Budapest, Hungary.

[2] S. Aimi, D. Lee, S. Sakai, and M. Sakuma, Classification of parabolic generating pairs of Kleinian groups with two parabolic generators, arXiv:2001.11662 v1. 
[3] G. Burde and H. Zieschang, Knots, de Gruyter Studies in Mathematics, 5, Walter de Gruyter, 2003.

[4] J. Scott Carter, A survey of quandle Ideas, Introductory Lectures on Knot theory, pp. 22-53 (2011).

[5] J. Cho, Optimistic limit of the colored Jones polynomial and the existence of a solution, Proceedings of the American Mathematical Society 144 (4) (2016) 1803-1814.

[6] J. Cho, Optimistic limit of the colored Jones polynomial and the complex volumes of hyperbolic links, J. Aust. Math. Soc. 100(2016), 303-337.

[7] J. Cho and J. Murakami, Optimistic limit of the colored Jones polynomial, Journal of Korean Mathematical Society 50 (3) (2013) 641-693.

[8] J. Cho, S. Yoon, C. K. Zickert, On the Hikami-Inoue conjecture, to appear in Algebr. Geom. Topol.

[9] Y. Cho, S. Kim, H. Kim, S. Yoon, Parabolic representations by knot diagram: computation up to 11 crossings, in preparation.

[10] J. H. Conway, An enumeration of knots and links and some of their algebraic properties, Proceedings of the conference on Computational problems in Abstract Algebra held at Oxford in 1967, J. Leech ed., (First edition 1970), Pergamon Press, 329-358.

[11] Peter R. Cromwell, Knots and links, cambridge University Press, 2004.

[12] S. Garrabrant, J. Hoste and P. Shanahan, Upper bounds in the Ohtsuki-Riley-Sakuma partial order on 2-bridge knots, J. Knot Theory Ramifications 21 (9), 1250084 (2012) [24 pages]

[13] S. Garoufalidis, D. P. Thurston, C. K. Zickert, The complex volume of $S L(n, \mathbb{C})$-representations of 3-manifolds, Duke Mathematical Journal, 164 (11) (2015) 2099-2160.

[14] S. Gukov, Three-Dimensional Quantum Gravity, Chern-Simons Theory, and the A-Polynomial, Commun. Math. Phys. 255, 577-627 (2005).

[15] M. Hirasawa and K. Murasugi, Twisted Alexander polynomials of 2-bridge knots associated to dihedral representations, J. Knot Theory Ramifications 27, No. 2 (2018) 1850015 (16 pages).

[16] H. J. Hsiao, On factorization of Chebyshev's polynomials of the first kind, Bulletin of the Institute of Mathematics, Academia Sinica 12 (1), 1984, pp. 89-94.

[17] J. Hoste and P. Shanahan, Trace fields of twist knots, J. Knot Theory Ramifications 10, No. 4 (2001), 625-639.

[18] A. Inoue and Y. Kabaya, Quandle homology and complex volume, Geometriae Dedicata 171, Issue 1, (2014), 265-292.

[19] D. Joyce, A classifying invariant of knots, the knot quandle, J. Pure Apll. Algebra, 23 (1982), 37-65.

[20] L. H. Kauffman and S. Lambropoulou, On the classification of rational tangles, Advances in Applied Mathematics, Volume 33, Issue 2, August 2004, 199-237.

[21] A. Kawauchi, A survey of knot theory, Birkhauser Verlag, Berlin, 1996.

[22] H. Kim, S. Kim, S. Yoon, Octahedral developing of knot complemenet I: pseudo-hyperbolic structure, Geometriae Dedicata, 197 (1), (2018) 123-172.

[23] H. Kim, S. Kim, S. Yoon, Octahedral developing of knot complemenet II: Ptolemy coordinate and its applications, arXiv:1904.06622 v1.

[24] T. Kitano and T. Morifuji, A note on Riley polynomials of 2-bridge knots, arXiv:math/1609.07819.

[25] M. L. Macasieb, K. L. Petersen, R. M. van Luijk, On character varieties of 2-bridge knot groups, Proc. London Math. Soc. 103(3) (2011), 473-504.

[26] C. Maclachlan and A. W. Reid, The Arithmetic of hyperbolic 3 manifolds, Springer (2003).

[27] H. Murakami and Y. Yokota, Volume Conjecture for Knots, Springer Briefs in Mathematical Physics 30, (2018).

[28] F. Nagasato, M. Suzuki and A. Tran, On minimality of two-bridge knots, Internat. J. Math. Vol. 28, No. 03, 1750020 (2017).

[29] E. A. Navas and S. Nelson, On symplectic quandles, Osaka J. Math. 45 (2008), 973-985.

[30] Walter D. Neumann, Extended Bloch group and the CheegerChernSimons class, Geom. Topol. Volume 8, Number 1 (2004), 413-474.

[31] T. Ohtsuki, R. Riley and M. Sakuma, Epimorphisms between 2-bridge link groups, Geom. Topol. Monogr. 14 (2008), 417-450. 
[32] R. Riley, Parabolic representations of knot groups, I, Proc. London Math. Soc. (3) 24 (1972), 217-242.

[33] R. Riley, Nonabelian representations of 2-bridge knot groups, Quart. J. Math. Oxford, 35(2) (1984), 191-208.

[34] Theodore J. Rivlin, Chebyshev polynomials. From Approximation Theory to Algebra and Number Theory, second edition, Pure Appl. Math. (New York), John Wiley \& Sons, Inc., New York, 1990.

[35] Henry Segerman, Stephan Tillmann, Pseudo-developing maps for ideal triangulations I: essential edges and generalised hyperbolic gluing equations, Topology and geometry in dimension three: Triangulations, Invariants, and Geometric Structures (Oklahoma, 2010), Contemp. Math. 560 (2011), 85102.

[36] M. Suzuki, Epimorphisms between 2-bridge knot groups and their crossing numbers, Algebraic \& Geometric Topology 17 (2017) 2413-2428.

[37] M. Yamagishi, A note on Chebyshev polynomials, cyclotomic polynomials and twin primes, Journal of Number theory 133 (2013), 2455-2463.

[38] D. N. Yetter, Quandles and monodromy, J. Knot Theory Ramifications 12 (2003), no. 4, pp. 523-541.

[39] Y. Yokota, On the complex volume of hyperbolic knots, J. Knot Theory Ramifications 20, No. 7 (2011), 955-976.

[40] C. K. Zickert, The volume and Chern-Simons invariant of a representation, Duke Mathematical Journal, 150 (3) (2009) 489-532.

Division of Liberal Arts and sciences, Mokpo National Maritime University, Mokpo, Chonnam, 530-729, Korea

E-mail address: khjo@mmu.ac.kr

Department of Mathematical Sciences, Seoul National University, Seoul, 08826, Korea

E-mail address: hyukkim@snu.ac.kr 